\title{
A New Continuum Model considering Driving Behaviors and Electronic Throttle Effect on a Gradient Highway
}

\author{
Yulei Jiao $\mathbb{D}^{1,2,3}$ Rongjun Cheng $\mathbb{D}^{1,2,3}$ and Hongxia Ge $\mathbb{D}^{1,2,3}$ \\ ${ }^{1}$ Faculty of Maritime and Transportation, Ningbo University, Ningbo 315211, China \\ ${ }^{2}$ Jiangsu Province Collaborative Innovation Center for Modern Urban Traffic Technologies, Nanjing 210096, China \\ ${ }^{3}$ National Traffic Management Engineering and Technology Research Centre Ningbo University Sub-Centre, \\ Ningbo 315211, China \\ Correspondence should be addressed to Rongjun Cheng; chengrongjun76@126.com
}

Received 28 March 2020; Accepted 15 April 2020; Published 18 May 2020

Academic Editor: Yumin Cheng

Copyright (c) 2020 Yulei Jiao et al. This is an open access article distributed under the Creative Commons Attribution License, which permits unrestricted use, distribution, and reproduction in any medium, provided the original work is properly cited.

\begin{abstract}
In order to explore the potential impact of sloping road on traffic flow, an improved car-following model considering electronic throttle (ET) dynamics and driver's driving characteristics on slope is proposed. Based on the improved car-following model, a new continuum model is established through the conversion relationship between microscopic variables and macroscopic variables. Firstly, the stability condition of the model is obtained by using the linear stability theory, after that the evolution process of traffic flow density wave near the neutral stability curve is studied by using the nonlinear analysis method, and we also get the improved KdV-Burgers equation. At the same time, numerical experiments and experimental verification of the model are carried out; the theoretical analysis and numerical results show that the ET effect and aggressive driving of drivers play an important role in alleviating traffic congestion to a certain extent.
\end{abstract}

\section{Introduction}

Nowadays, transportation has become an important part of people's economic life and plays a crucial role in the development of society economy and the improvement of people's life satisfaction. However, with the development of urbanization and travel motorization, traffic congestion is becoming more and more serious. The impact of urban traffic congestion on society and individual is mainly manifested in economic losses, environmental pollution, and potential safety hazards. How to solve the problem of traffic congestion has become an urgent problem for city managers. Therefore, more and more experts and scholars turn their attention to the research field of traffic flow control [1-9].

To solve the problem of traffic congestion, it is necessary to understand the evolution disciplinarian of traffic flow. In order to deeply research the traffic flow, the famous British scholars Lighthill and Whitharn $[10,11]$ proposed the Fluid-Dynamical Theories in 1955, which simulated the traffic flow as fluid and established the continuity equation of traffic flow by using the basic principles of hydrodynamics:

$$
\frac{\partial \rho}{\partial t}+\frac{\partial(\rho v)}{\partial x}=0,
$$

where $\rho, v, t$, and $x$ represent the density, velocity, time, and spatial position, respectively.

The relationship among flow rate, velocity, and density of traffic flow is deduced from the theory, and then the mathematical model describing the characteristics of traffic flow evolution is established. Since the LWR model was put forward, the research on the relationship of basic parameters of traffic flow has been carried out for decades, and many models have been put forward, which can be roughly divided into three categories: car-following model [12-27], lattice hydrodynamic model [28-37], and continuum model [38-46]. These models have important application value, which are the basis for defining the service level of traffic service facilities, and they can estimate the traffic capacity 
and operation state when the traffic capacity is reached, so they have been widely used in traffic planning.

However, most traffic flow scholars believe that the speed assumed in the LWR model is always the equilibrium speed, which cannot be used to simulate various non-equilibrium traffic phenomena, such as the stop-and-go traffic, etc. To solve this problem, the researchers proposed a higher-order model. In 1971, Payne [47] proposed the first higher-order model:

$$
\frac{\partial v}{\partial t}+v \frac{\partial v}{\partial x}=-\frac{a^{\prime}}{\rho T} \frac{\partial \rho}{\partial x}+\frac{v_{e}-v}{T},
$$

where $T$ is the relaxation time and $a l$ is the expected coefficient. In this model, the equilibrium velocity-density relationship is replaced by the kinetic equation of velocity, which allows the velocity to deviate from the equilibrium velocity.

In the field of traffic physics, the optimization speed model has been widely concerned because of its eximious mathematical and physical properties, such as being analyzable and easy to simulate. This is the optimal velocity (OV) model proposed by Bando et al. [8, 48] in 1995, expressed as follows:

$$
\frac{\mathrm{d} v_{n}(t)}{\mathrm{d} t}=a\left[V\left(\Delta x_{n}(t)\right)-v_{n}(t)\right]
$$

where $a$ is the sensitivity coefficient, which is regarded as the reciprocal of the driver's reaction time T and $V\left(\Delta x_{n}(t)\right)$ is the optimization speed function, which is monotonically increasing and has an upper bound.

After the OV model was proposed, it received extensive attention and research in the field of transportation, especially in the field of traffic physics. On account of the OV model, various improved models were proposed. Tilch and Helbing [49] used the measured traffic data to identify the parameters of the OV model and found that, in the OV model, vehicle acceleration was too large, the deceleration was unreasonable, and crash would occur. They believed that when the current car's speed is less than that of the rear car, the influence of the speed difference should be considered. Thus, a general force model (GF) is proposed:

$$
\frac{\mathrm{d} v_{n}(t)}{\mathrm{d} t}=a\left[V\left(\Delta x_{n}(t)\right)-v_{n}(t)\right]+\lambda H\left(-\Delta v_{n}(t)\right) \Delta v_{n}(t),
$$

where $H(\cdot)$ is Heaviside function and $\lambda$ is the sensitivity coefficient,

$$
H(x)= \begin{cases}0, & x \leq 0, \\ 1, & x>0 .\end{cases}
$$

Jiang et al. [50] used GF model to simulate the starting process of a stationary motorcade, and they found that the starting wave speed of the traffic flow in this model was too low. They believe that not only should the influence of the speed difference be considered when the speed of the front car is less than that of the rear car, but also the influence of the speed difference be considered when the speed of the front car is greater than that of the rear car. Based on this, they proposed a full velocity difference model (FVDM):

$$
\frac{\mathrm{d} v_{n}(t)}{\mathrm{d} t}=a\left[V\left(\Delta x_{n}(t)\right)-v_{n}(t)\right]+\lambda \Delta v_{n}(t) .
$$

Driver's driving behavior and vehicle running characteristics are the basis of traffic flow research. At the same time, the study of driver's driving behavior is a complex process, which has attracted extensive attention of scholars in recent years. Researchers have proposed many new models based on different perspectives of driver's driving behavior.

However, in the field of continuum models, there are few studies on the driving characteristics of drivers on slopes. Therefore, a new extended continuum model is proposed in this paper, which considers both the driver's driving characteristics and the "electronic throttle" effect on the slope. Firstly, this paper analyzes the impact of driver's aggressive driving and timid driving characteristics on traffic flow, establishes a new car-following model based on driver's driving behavior in slope environment, and obtains a new extended continuum model through the transformation relationship between macrovariables and microvariables. The stability of traffic flow under the influence of driver characteristics and electronic throttle angle in different gradient environments is discussed through the linear analysis. The standard KdV-Burgers equation and solitary wave solution of the equation are derived by nonlinear stability analysis near the neutral stability curve. Then, through the numerical simulation experiments of density waves, shock waves, and rarefaction waves, the effects of slope, driver's driving characteristics, and "electronic throttle" effect on traffic flow parameters such as speed, acceleration, and traffic density are studied. Finally, a summary of the research contents in this paper is given.

\section{The Continuum Model and Nonlinear Analysis}

It is well known that timid drivers who lack proficiency ability in driving will choose to drive cautiously, while those who have proficiency ability in driving will choose aggressive driving to get the best speed they want. Based on this, considering the driver's timid and aggressive driving psychology, Cheng [51] proposed an extended continuum model that takes into account the effects of driver's timid and aggressive attributions:

$$
\begin{aligned}
\frac{\mathrm{d} v_{n}(t)}{\mathrm{d} t}= & a\left[p V\left(\Delta x_{n}(t+\alpha \tau)\right)+(1-p) V\left(\Delta x_{n}(t-\alpha \tau)\right)\right. \\
& \left.-v_{n}(t)\right]+\lambda \Delta v_{n}(t)
\end{aligned}
$$

where $\Delta x_{n}=x_{n+1}-x_{n}, \Delta v_{n}=v_{n+1}-v_{n}, a=1 / \tau, a$ is the driver's sensitivity coefficient, $\alpha \tau$ represents an additional reaction time and $\alpha$ is a variable parameter, $\lambda$ is the driver's sensitivity coefficient to speed difference, and $0 \leq p \leq 1$ 
represents the strength influence factor of the driver's characteristics. When $0.5<p<1$, aggressive driving behavior will be dominant; on the contrary, when $0<p<0.5$, the influence of timid driving was greater.

The first-order Taylor expansion of the micro-variables $\Delta x_{n}(t \pm \alpha \tau)$ is carried out, while the higher-order term is neglected, and the results are as follows:

$$
\begin{aligned}
& \Delta x_{n}(t+\alpha \tau)=\Delta x_{n}(t)+\frac{\mathrm{d} \Delta x_{n}(t)}{\mathrm{d} t} \alpha \tau=\Delta x_{n}(t)+\alpha \tau \Delta v_{n}(t), \\
& \Delta x_{n}(t-\alpha \tau)=\Delta x_{n}(t)-\frac{\mathrm{d} \Delta x_{n}(t)}{\mathrm{d} t} \alpha \tau=\Delta x_{n}(t)-\alpha \tau \Delta v_{n}(t) .
\end{aligned}
$$

Based on the Taylor expansion mentioned above, we can get

$$
\begin{aligned}
V\left(\Delta x_{n}(t+\alpha \tau)\right)= & V\left(\Delta x_{n}(t)+\alpha \tau \Delta v_{n}(t)\right)=V\left(\Delta x_{n}(t)\right) \\
& +\alpha \tau \Delta v_{n}(t) V^{\prime}\left(\Delta x_{n}(t)\right), \\
V\left(\Delta x_{n}(t-\alpha \tau)\right)= & V\left(\Delta x_{n}(t)-\alpha \tau \Delta v_{n}(t)\right)=V\left(\Delta x_{n}(t)\right) \\
& -\alpha \tau \Delta v_{n}(t) V^{\prime}\left(\Delta x_{n}(t)\right) .
\end{aligned}
$$

In previous studies, most of the models only considered the situation of flat roads. However, in real life, due to the change of terrain, roads cannot always be flat, such as mountain roads. On the other hand, according to the actual traffic demand, roads will be built in the form of uphill and downhill, such as the approach part of overpasses. So, what is the difference between traffic flow on sloping roads and that on flat roads? How to analyze the car-following behavior on sloping highway is a realistic problem. In recent years, in the theory of carfollowing, the slope factor has also been considered by researchers. For example, Yang et al. [52] gave a car-following model on the slope to study the effects of the road's slope on the electricity consumption, Zhou et al. [53] came up with a generalized car-following model of relative speed on slope, and considering the influence of gravity, Chen et al. [54] proposed a generalized model with consideration of the gravitational force. However, the above models do not consider the driver's driving characteristics and the influence of electronic throttle effect on the stability of traffic flow. In practice, the driver's driving characteristics and electronic throttle effect play an important role in traffic flow. Therefore, these two factors cannot be ignored when establishing traffic flow model.

The following is an extended model of the relative velocity of traffic flow on a gradient road proposed by Zhou et al. [53] in 2014,

$$
\frac{\mathrm{d}^{2} x_{n}(t)}{\mathrm{d} t^{2}}=a\left[V\left(\Delta x_{n}(t)\right)-\frac{\mathrm{d} x_{n}(t)}{\mathrm{d} t}\right]+\lambda a \Delta v_{n}(t),
$$

with

$$
\begin{aligned}
V\left(\Delta x_{n}(t)\right) & =\frac{v_{f, \text { max }} \mp v_{g, \text { max }}}{2}\left[\tanh \left(\Delta x_{n}-h_{c, \theta}\right)+\tanh \left(h_{c, \theta}\right)\right], \\
h_{c, \theta} & =h_{c}(1 \mp \sin \theta),
\end{aligned}
$$

where $v_{f, \max }$ represents the maximum allowable speed of a vehicle on a road with no gradient, $v_{g, \max }=m g \sin \theta / \mu$, $v_{g, \max }$ is proportional to slope $\sin \theta$, where $\mu$ is the coefficient of friction, $\theta$ is slope, $h_{c}$ is a safe distance without slope, and $h_{c, \theta}$ is the safe distance on the slope.

Based on the existing traffic flow models, considering the effects of driver's characteristics and electronic throttle effect on traffic flow, this chapter proposes a traffic flow following model on sloping roads.

$$
\begin{aligned}
\frac{\mathrm{d} v_{n}(t)}{\mathrm{d} t}= & a\left[p V\left(\Delta x_{n}(t+\alpha \tau)\right)+(1-p) V\left(\Delta x_{n}(t-\alpha \tau)\right)-v_{n}(t)\right] \\
& +\lambda a \Delta v_{n}(t)+\beta \Delta \varphi_{n}(t)
\end{aligned}
$$

where $\beta$ is the sensitivity coefficient of angle difference $\Delta \phi_{n}(t)$ for electronic throttle.

In addition, the relationship among the electronic throttle opining angle, acceleration, and speed of the car can be expressed as follows [55]:

$$
\frac{\mathrm{d} v_{n}(t)}{\mathrm{d} t}=-b\left(v_{n}(t)-v_{0}\right)+m \bar{\phi}_{n}+h_{n},
$$

where the initial electronic throttle opining angle and speed are $\phi_{0}$ and $v_{0}, \bar{\phi}_{n}=\phi_{n}-\phi_{0}$ is the angle difference of the electronic throttle, $b$ and $m$ change with the $v_{0}$ changes, and $h_{n}$ represents the disturbance of nonmodelled dynamics influence.

On the foundation of equation (13), the formula of the ET opening angle difference between the car $n$ and car $n+1$ can be expressed as follows:

$$
\Delta \phi_{n}=\phi_{n+1}-\phi_{n}=\frac{1}{m}\left[\frac{\mathrm{d} v_{n+1}(t)}{\mathrm{d} t}-\frac{\mathrm{d} v_{n}(t)}{\mathrm{d} t}+b \Delta v_{n}(t)\right] .
$$

Equation (14) is introduced into equation (12) and the following formula is obtained:

$$
\begin{aligned}
\frac{\mathrm{d} v_{n}(t)}{\mathrm{d} t}= & \frac{m}{m+\beta} a\left[V\left(\Delta x_{n}(t)\right)-v_{n}(t)\right]+\frac{\beta}{m+\beta} \frac{\mathrm{d} v_{n+1}(t)}{\mathrm{d} t} \\
& +\frac{m}{m+\beta}\left[a(2 p-1) \alpha \tau V_{\prime}\left(\Delta x_{n}(t)\right)+\lambda a+\frac{b \beta}{m}\right] \Delta v_{n}(t) .
\end{aligned}
$$

In order to study the influence of the vehicle dynamics information in the stability of traffic flow, we convert discrete variables into continuous variables though the following relations:

$$
\begin{aligned}
v_{n}(t) & \longrightarrow v(x, t), \\
v_{n+1}(t) & \longrightarrow v(x+\Delta, t), \\
V\left(\Delta x_{n}(t)\right) & \longrightarrow \frac{v_{f, \text { max }} \mp v_{g, \text { max }}}{2} V_{e}(\rho), \\
V_{\prime}\left(\Delta x_{n}(t)\right) & \longrightarrow V^{\prime}(h(c, \theta)) \longrightarrow \frac{v_{f, \text { max }} \mp v_{g, \text { max }}}{2} V_{e}^{\prime}(\rho),
\end{aligned}
$$


where the space between the two cars is expressed as $\Delta$ and $\rho(x, t)$ and $v(x, t)$ represent the macroscopic density and macroscopic velocity, respectively. The average headway is expressed as $h=1 / \rho, V_{e}(\rho)$ means the equilibrium speed, and $\bar{V}^{\prime}(h)=-\rho^{2} V_{e}^{\prime}(\rho)$.
After a simple analysis, we develop the Taylor series expansion for $v(x+\Delta, t)$ and get the following formula after ignoring the higher-order terms:

$$
\begin{aligned}
\Delta v_{n}(t) & =v(x+\Delta, t)-v(x, t)=v \prime(x, t) \Delta+\frac{1}{2} v^{\prime \prime}(x, t) \Delta^{2}, \\
\frac{\mathrm{d} v_{n+1}(t)}{\mathrm{d} t} & =\frac{\mathrm{d} v(x+\Delta, t)}{\mathrm{d} t}=\frac{\partial v(x, t)}{\partial x} v(x+\Delta, t)+\frac{\partial v(x+\Delta, t)}{\partial t} \\
& =v_{x}\left(v+\Delta v_{x}\right)+\frac{\partial\left(v+\Delta v_{x}\right)}{\partial t}=v v_{x}+\Delta v_{x} v_{x}+v_{t}+\Delta v_{x t} .
\end{aligned}
$$

The new extended continuum model is obtained by transforming micro-variables into macro-variables and sorting them out as follows:

$$
\begin{aligned}
v_{t}+\left\{v-\frac{\beta}{m} \Delta v_{x}-\left[\lambda a+\frac{b \beta}{m}-a(2 p-1) \alpha \tau \rho^{2} \frac{4 \mp \sin \theta}{2} V_{e}^{\prime}(\rho)\right] \Delta\right\} v_{x} \\
=a\left[\frac{4 \mp \sin \theta}{2} V_{e}^{\prime}(\rho)-v(x, t)\right]+\frac{\beta}{m} \Delta v_{x t} \\
+\left[\lambda a+\frac{b \beta}{m}-a(2 p-1) \alpha \tau \rho^{2} \frac{4 \mp \sin \theta}{2} V_{e}^{\prime}(\rho)\right] \frac{1}{2} \Delta^{2} v_{x x} .
\end{aligned}
$$

\section{Linear Stability Analysis}

In order to do the qualitative analysis of the extended new model, this paper adopts the linear stability analysis method. All vehicles are assumed to have the same speed and headway in the steady state. Then, based on the basic idea of the fluid dynamics simulation theory of traffic flow proposed by Richards et al. [56, 57], the traffic flow is analogized to a fluid, and its continuity fluid dynamics equation is established to study.

$$
\left\{\begin{array}{l}
\frac{\partial \rho}{\partial \mathrm{t}}+\rho \frac{\partial v}{\partial x}+v \frac{\partial \rho}{\partial x}=0 \\
\frac{\partial v}{\partial t}+\left[v-\frac{\beta}{m} \Delta \frac{\partial v}{\partial x}-\left(\lambda a+\frac{b \beta}{m}-a(2 p-1) \alpha \tau \rho^{2} \frac{4 \mp \sin \theta}{2} V_{e}^{\prime}(\rho)\right) \Delta\right] \frac{\partial v}{\partial x} \\
=a\left[\frac{4 \mp \sin \theta}{2} V_{e}(\rho)-v\right]+\frac{\beta}{m} \Delta v_{x t}+\left[\lambda a+\frac{b \beta}{m}-a(2 p-1) \alpha \tau \rho^{2} \frac{4 \mp \sin \theta}{2} V_{e}^{\prime}(\rho)\right] \frac{\Delta^{2}}{2} v_{x x} .
\end{array}\right.
$$


In order to facilitate the study of linear analysis, the where equations are rewritten into matrices as follows:

$$
\frac{\partial \vec{U}}{\partial t}+\vec{A} \frac{\partial \vec{U}}{\partial x}=\vec{E}
$$

$$
\begin{aligned}
& \vec{U}=\left[\begin{array}{l}
\rho \\
v
\end{array}\right], \\
& \vec{A}=\left[\begin{array}{c}
v \\
0 \quad v-\frac{\beta}{m} \Delta v_{x}-\left[\lambda a+\frac{b \beta}{m}-a(2 p-1) \alpha \tau \rho^{2} \frac{4 \mp \sin \theta}{2} V_{e}^{\prime}(\rho)\right] \Delta
\end{array}\right], \\
& \overrightarrow{\mathrm{E}}=\left(\begin{array}{c}
0, \\
a\left[\frac{4 \mp \sin \theta}{2} V_{e}^{\prime}(\rho)-v(x, t)\right]+\frac{\beta}{m} \Delta v_{x t} \\
+\left[\lambda a+\frac{b \beta}{m}-a(2 p-1) \alpha \tau \rho^{2} \frac{4 \mp \sin \theta}{2} V_{e}^{\prime}(\rho)\right] \frac{1}{2} \Delta^{2} v_{x x}
\end{array}\right),
\end{aligned}
$$

and the eigenvalues of $\vec{A}$ are

$$
\begin{aligned}
\lambda_{1}= & v, \\
\lambda_{2}= & a\left[\frac{4 \mp \sin \theta}{2} V_{e}^{\prime}(\rho)-v(x, t)\right]+\frac{\beta}{m} \Delta v_{x t} \\
& +\left[\lambda a+\frac{b \beta}{m}-a(2 p-1) \alpha \tau \rho^{2} \frac{4 \mp \sin \theta}{2} V_{e}^{\prime}(\rho)\right] \frac{1}{2} \Delta^{2} v_{x x} .
\end{aligned}
$$

Through the research and calculation, it is obvious to find that the macro traffic flow velocity $v$ is larger than the characteristic velocity. The phenomenon proves that the proposed extension of the traffic flow model satisfies the anisotropy.

Traffic congestion is a phenomenon of unstable traffic flow. Small disturbances caused by human or objective factors will diffuse upward along the traffic flow, making the original smooth traffic flow gradually evolve into traffic congestion, resulting in the instability of the traffic flow system. If the small disturbance can be gradually reduced and disappear in the process of propagation, or controlled in a certain small range, then the vehicles in the traffic flow system can run smoothly, thereby achieving the purpose of controlling traffic congestion.

It is assumed that the traffic flow system is uniform at the beginning, and the constants $\rho_{0}$ and $v_{0}$ represent the initial density and the initial velocity in the uniform state. As a result, the expression of the steady-state solution of uniform flow is

$$
\begin{aligned}
& \rho(x, t)=\rho_{0}, \\
& v(x, t)=v_{0},
\end{aligned}
$$

and then disturbance is added to observe the change of the traffic density. The expression is as follows:

$$
\left(\begin{array}{c}
\rho(x, t) \\
v(x, t)
\end{array}\right)=\left(\begin{array}{c}
\rho_{0} \\
v_{0}
\end{array}\right)+\left(\begin{array}{c}
\hat{\rho}_{k} \\
\widehat{v}_{k}
\end{array}\right) \exp \left(i k x+\sigma_{k} t\right) .
$$

Combining equations (19) and (23) and ignoring the nonlinear higher-order terms, we get

$$
\begin{aligned}
& \left(\sigma_{k}+v_{0} i k\right) \widehat{\rho}_{k}+\rho_{0} i k \widehat{v}_{k}=0, \\
& a \frac{4 \mp \sin \theta}{2} V_{e}^{\prime}\left(\rho_{0}\right) \widehat{\rho}_{k}-\frac{\Delta^{2} k^{2}}{2}\left[\lambda a+\frac{b \beta}{m}-a(2 p-1) \alpha \tau \rho_{0}^{2} \frac{4 \mp \sin \theta}{2} V_{e}^{\prime}\left(\rho_{0}\right)\right] \widehat{v}_{k} \\
& \quad-\left[v_{0}-\left(\lambda a+\frac{b \beta}{m}-a(2 p-1) \alpha \tau \rho_{0}^{2} \frac{4 \mp \sin \theta}{2} V_{e}^{\prime}\left(\rho_{0}\right)\right) \Delta\right] i k \widehat{v}_{k}+\left(\frac{\beta}{m} \Delta i k \sigma_{k}-a-\sigma_{k}\right) \widehat{v}_{k}=0 .
\end{aligned}
$$


The equations are rewritten into matrices as follows:

$$
\begin{aligned}
\vec{C} \cdot \vec{X} & =\vec{B}, \\
\vec{X} & =\left(\begin{array}{c}
\widehat{\rho}_{k} \\
\hat{v}_{k}
\end{array}\right), \\
\vec{B} & =\left(\begin{array}{c}
0 \\
0
\end{array}\right), \\
\vec{C} & =\left(\begin{array}{c}
\sigma_{k}+i k v_{0} i k \rho_{0}-\frac{\Delta^{2} k^{2}}{2}\left[\lambda a+\frac{b \beta}{m}-a(2 p-1) \alpha \tau \rho_{0}^{2} \frac{4 \mp \sin \theta}{2} V_{e}^{\prime}\left(\rho_{0}\right)\right] \\
a \frac{4 \mp \sin \theta}{2} V_{e}^{\prime}\left(\rho_{0}\right)-\left[v_{0}-\left(\lambda a+\frac{b \beta}{m}-a(2 p-1) \alpha \tau \rho_{0}^{2} \frac{4 \mp \sin \theta}{2} V_{e}^{\prime}\left(\rho_{0}\right)\right) \Delta\right] i k
\end{array}\right) .
\end{aligned}
$$

Regarding $\widehat{\rho}_{k}$ and $\widehat{v}_{k}$ as the unknown parameters in the equations, then we will get $\sigma_{k}$ that satisfies the following quadratic equation:

$$
\begin{aligned}
& \left(\sigma_{k}+i k v_{0}\right)\left\{-\frac{\Delta^{2} k^{2}}{2}\left[\lambda a+\frac{b \beta}{m}-a(2 p-1) \alpha \tau \rho_{0}^{2} \frac{4 \mp \sin \theta}{2} V_{e}^{\prime}\left(\rho_{0}\right)\right]\right. \\
& \left.-\left[v_{0}-\left(\lambda a+\frac{b \beta}{m}-a(2 p-1) \alpha \tau \rho_{0}^{2} \frac{4 \mp \sin \theta}{2} V_{e}^{\prime}\left(\rho_{0}\right)\right) \Delta\right] i k+\left(\frac{\beta}{m} \Delta i k \sigma_{k}-a-\sigma_{k}\right)\right\}-a \frac{4 \mp \sin \theta}{2} V_{e}^{\prime}\left(\rho_{0}\right) i k \rho_{0}=0 .
\end{aligned}
$$

According to the control theory of stability criterion, the stability condition of traffic flow is obtained. Therefore, in terms of the neutral stability of stable well, we get

$$
a_{s}=\frac{\rho_{0}(4 \mp \sin \theta / 2 \Delta) V_{e}^{\prime}\left(\rho_{0}\right)+(b \beta / m)}{(2 p-1) \alpha \tau \rho_{0}^{2}(4 \mp \sin \theta / 2) V_{e}^{\prime}\left(\rho_{0}\right)-\lambda} .
$$

Performing the Taylor expansion for $\sigma_{k}$, we will get

$$
\operatorname{Im}\left(\sigma_{k}\right) \approx-a\left[v_{0}+\rho_{0} \frac{4 \mp \sin \theta}{2 \Delta} V_{e}^{\prime}\left(\rho_{0}\right)\right]+o\left(k^{3}\right) .
$$

According to equation (29), we infer that

$$
c\left(\rho_{0}\right)=v_{0}+\rho_{0} \frac{4 \mp \sin \theta}{2 \Delta} V_{e}^{\prime}\left(\rho_{0}\right)
$$

this is the same as that of Jiang Rui-Wu Qingsong's velocity gradient model [58] and modified model.

Figures 1 and 2 correspond to the phase diagrams of parameter space $(\rho, a)$ of the newly proposed model in the uphill and downhill road environments. The parameters are $\lambda=0.5, \Delta=10 \mathrm{~m}, \tau=0.5 \mathrm{~s}, \alpha=0.8, m=5, b=0.5, \beta=1.2$ when considering the influence of electronic throttle angle, $p=0.8$ when considering driver's aggressive driving, and $p=0.2$ when considering driver's timid driving, and the neutral stability lines of drivers in different slope $\theta=0,2,4,6$ situations are simulated, respectively. The parametric space $(\rho, a)$ is divided into two parts by the neutral stability curve: the instability region below the curve and the stability region above the curve.

In Figure 1, the stability region becomes larger and larger with the increase of slope $\theta$ on the uphill slope; similarly, in Figure 2, the stability region becomes smaller and smaller with the increase of slope $\theta$ on the downhill slope. Further, comparing the lower part of the neutral stability curve of the model with different driver's characteristics in the uphill and downhill scenarios, we can see that the unstable area of aggressive driving is smaller than that of timid driving, which indicates that the traffic flow of aggressive driving is more stable than that of timid driving. Similarly, in the uphill and downhill scenarios, the stability region considering the influence of electronic throttle angle is larger than that without considering the influence of electronic throttle angle, which indicates that considering the influence of electronic throttle angle will make traffic flow more stable. It can be concluded that when the uphill slope is larger, the traffic flow is more stable, and the driver's aggressive driving helps to improve the stability of the traffic flow; when the downhill slope is smaller, the traffic flow is more stable, and the driver's aggressive driving helps to improve the stability of the traffic flow. At the same time, considering the electronic throttle factor can effectively restrain traffic congestion. 

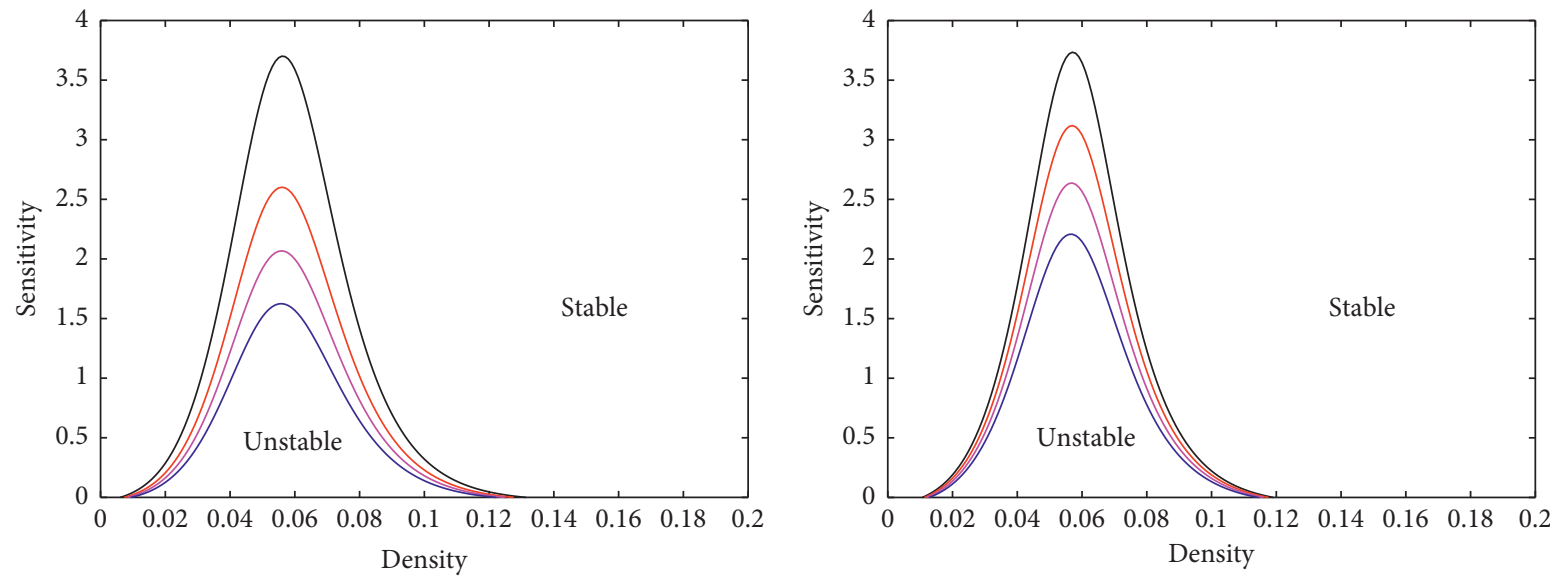

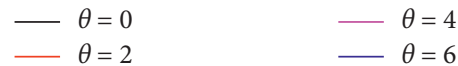

(a)

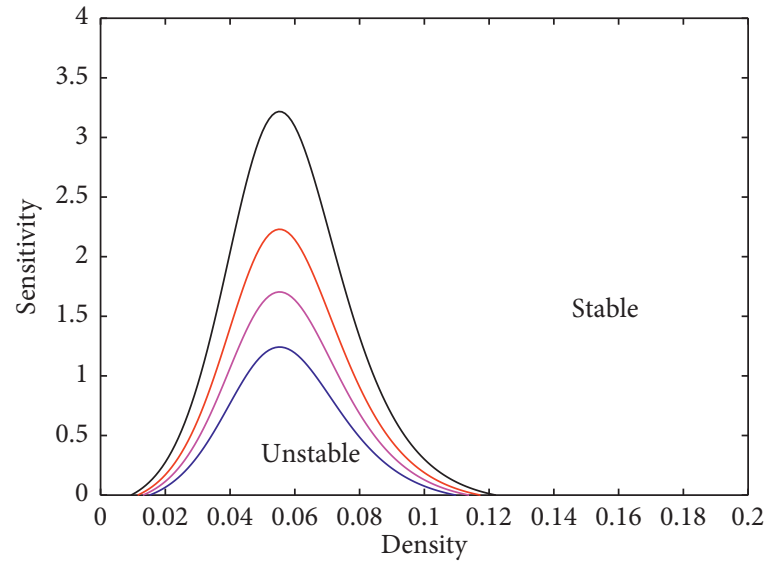

(c)

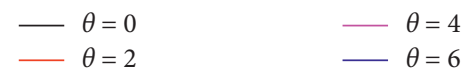

(b)

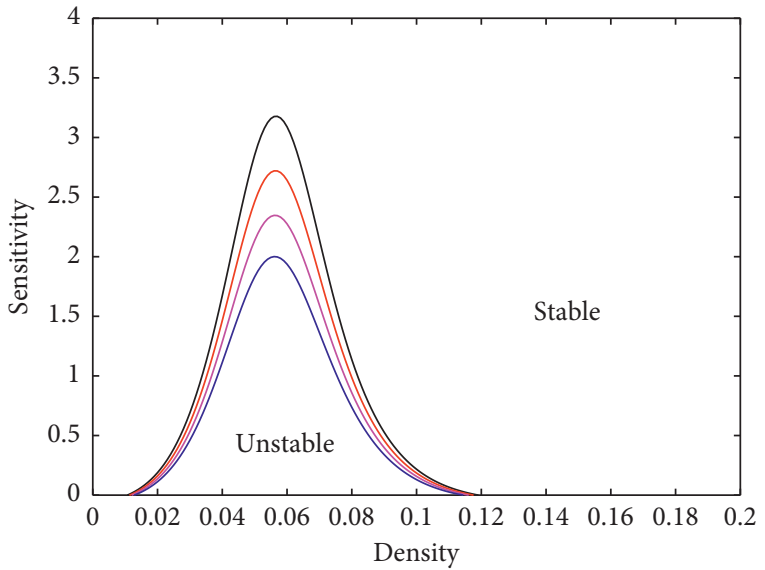

(d)

FIgUre 1: The neutral stability curves in uphill situation. (a) Aggressive driving without ET. (b) Timid driving without ET. (c) Aggressive driving with ET. (d) Timid driving with ET.

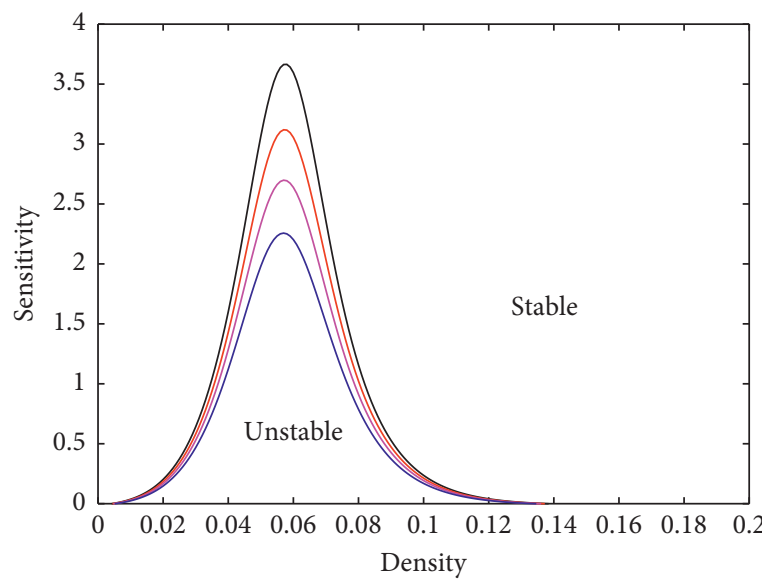

$\begin{aligned} \theta & =0 \\ -\theta & =2\end{aligned}$

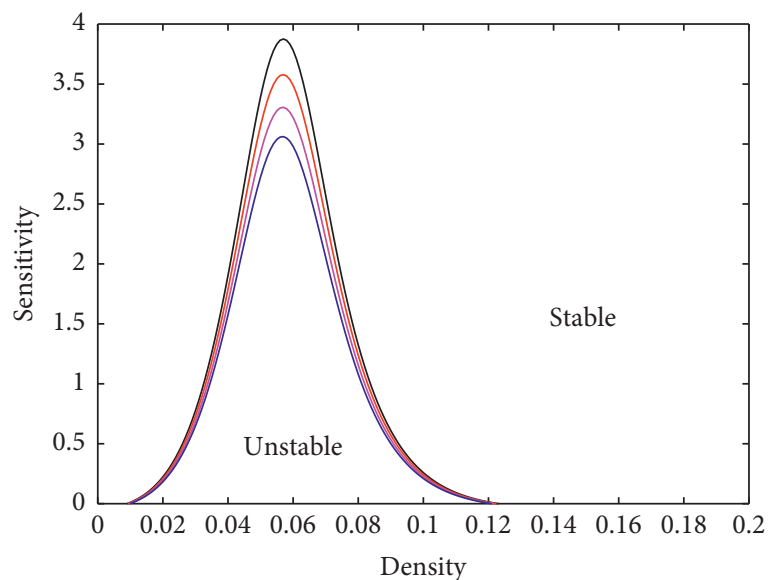

$\theta=0$
$-\theta=2$

$\theta=4$

(a)

(b)

Figure 2: Continued. 


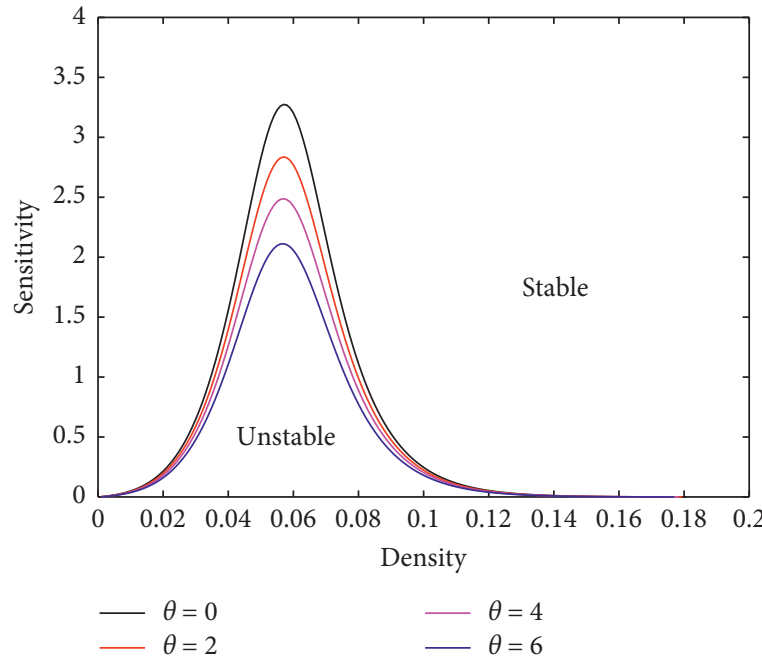

(c)

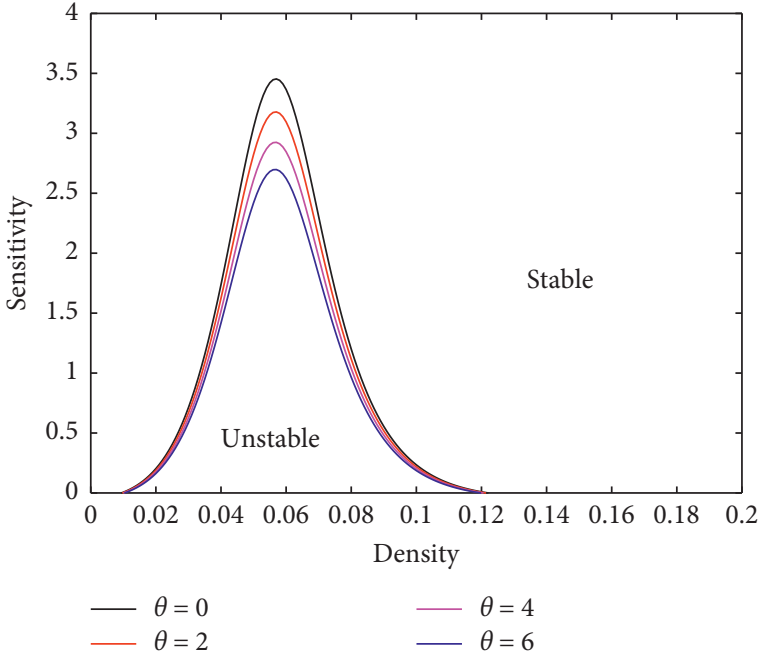

(d)

FIgURE 2: The neutral stability curves in downhill situation. (a) Aggressive driving without ET. (b) Timid driving without ET. (c) Aggressive driving with ET. (d) Timid driving with ET.

\section{Nonlinear Analysis}

For the sake of exploring the behavior of the system near the neutral stability, equation (19) is adopted to introduce a new coordinate system [38] as follows:

$$
z=x-c t
$$

The equation can be converted into the following formula:

$$
\left\{\begin{array}{l}
-c \rho_{z}+q_{z}=0 \\
-c v_{z}+v_{z}\left[v-\left(\lambda a+\frac{b \beta}{m}-a(2 p-1) \alpha \tau \rho^{2} \frac{4 \mp \sin \theta}{2} V_{e}^{\prime}(\rho)\right) \Delta-\frac{\beta}{m} \Delta v_{z}\right] \\
=a\left[\frac{4 \mp \sin \theta}{2} V_{e}(\rho)-v\right]+\left[\lambda a+\frac{b \beta}{m}-a(2 p-1) \alpha \tau \rho^{2} \frac{4 \mp \sin \theta}{2} V_{e}^{\prime}(\rho)\right] \frac{\Delta^{2}}{2} v_{z z}-c \frac{\beta}{m} \Delta v_{z z}
\end{array}\right.
$$

where $q=\rho v$ is the flow of traffic, which can be obtained from equation (31):

$$
\begin{aligned}
& v_{z}=\frac{c \rho_{z}}{\rho}-\frac{q \rho_{z}}{\rho^{2}}, \\
& v_{z z}=\frac{c \rho_{z z}}{\rho}-\frac{2 c \rho_{z}^{2}}{\rho^{2}}-\frac{q \rho_{z z}}{\rho^{2}}+\frac{2 q \rho_{z}^{2}}{\rho^{3}}+b_{2} \rho_{z},
\end{aligned}
$$

$$
\begin{aligned}
& -c\left(\frac{c \rho_{z}}{\rho}-\frac{q \rho_{z}}{\rho^{2}}\right)+\left(\frac{c \rho_{z}}{\rho}-\frac{q \rho_{z}}{\rho^{2}}\right)\left[\frac{q}{\rho}-\left(\lambda a+\frac{b \beta}{m}-a(2 p-1) \alpha \tau \frac{4 \mp \sin \theta}{2} V_{e}^{\prime}(\rho)\right) \Delta-\frac{\beta}{m} \Delta\left(\frac{c \rho_{z}}{\rho}-\frac{q \rho_{z}}{\rho^{2}}\right)\right] \\
= & a\left[\frac{4 \mp \sin \theta}{2} V_{e}(\rho)-\frac{q}{\rho}\right]+\left[\lambda a+\frac{b \beta}{m}-a(2 p-1) \alpha \tau \frac{4 \mp \sin \theta}{2} V_{e}^{\prime}(\rho)\right] \frac{\Delta^{2}}{2}\left(\frac{c \rho_{z z}}{\rho}-\frac{2 c \rho_{z}^{2}}{\rho^{2}}-\frac{q \rho_{z z}}{\rho^{2}}+\frac{2 q \rho_{z}^{2}}{\rho^{3}}\right) \\
& -c \frac{\beta}{m} \Delta\left(\frac{c \rho_{z z}}{\rho}-\frac{2 c \rho_{z}^{2}}{\rho^{2}}-\frac{q \rho_{z z}}{\rho^{2}}+\frac{2 q \rho_{z}^{2}}{\rho^{3}}\right) .
\end{aligned}
$$

Bringing equations (33) and (34) into equation (32), we can get 
The traffic flow $q$ is expanded as follows:

$$
q=\rho V_{e}(\rho)+b_{1} \rho_{z}+b_{2} \rho_{z} \text {. }
$$

$b_{1}$ and $b_{2}$ can be easily solved, because the traffic flow is stable and uniform. Inserting equation (36) into equation (35), we will receive

$$
\begin{aligned}
& -c\left(\frac{c \rho_{z}}{\rho}-\frac{V_{e}(\rho) \rho_{z}}{\rho}\right)+\left(\frac{c \rho_{z}}{\rho}-\frac{V_{e}(\rho) \rho_{z}}{\rho}\right)\left[V_{e}(\rho)-\frac{\beta}{m} \Delta\left(\frac{c \rho_{z}}{\rho}-\frac{V_{e}(\rho) \rho_{z}}{\rho}\right)\right] \\
& -\left(\frac{c \rho_{z}}{\rho}-\frac{V_{e}(\rho) \rho_{z}}{\rho}\right)\left(\lambda a+\frac{b \beta}{m}-a(2 p-1) \alpha \tau \frac{4 \mp \sin \theta}{2} V_{e}^{\prime}(\rho)\right) \Delta \\
= & a\left[\frac{4 \mp \sin \theta}{2} V_{e}(\rho)-V_{e}(\rho)-\frac{b_{1} \rho_{z}}{\rho}-\frac{b_{2} \rho_{z z}}{\rho}\right]-c \frac{\beta}{m} \Delta\left(\frac{c \rho_{z z}}{\rho}-\frac{2 c \rho_{Z}^{2}}{\rho^{2}}-\frac{V_{e}(\rho) \rho_{z z}}{\rho}+\frac{2 V_{e}(\rho) \rho_{z}^{2}}{\rho^{2}}\right) \\
& +\left[\lambda a+\frac{b \beta}{m}-a(2 p-1) \alpha \tau \frac{4 \mp \sin \theta}{2} V_{e}^{\prime}(\rho)\right] \frac{\Delta^{2}}{2}\left(\frac{c \rho_{z z}}{\rho}-\frac{2 c \rho_{Z}^{2}}{\rho^{2}}-\frac{V_{e}(\rho) \rho_{z z}}{\rho}+\frac{2 V_{e}(\rho) \rho_{z}^{2}}{\rho^{2}}\right) .
\end{aligned}
$$

According to equation (36), When balancing the coefficients of $\rho_{z}$ and $\rho_{z z}$ on both sides of the equation, the value of $b_{1}$ and $b_{2}$ can be obtained. Therefore, we get

$$
\left\{\begin{array}{l}
b_{1}=\frac{1}{a}\left(V_{e}(\rho)-c\right)\left\{V_{e}(\rho)-c-\left[\lambda a+\frac{b \beta}{m}-a(2 p-1) \alpha \tau \frac{4 \mp \sin \theta}{2} V_{e}^{\prime}(\rho)\right] \Delta\right\}, \\
b_{2}=-\frac{1}{a}\left(V_{e}(\rho)-c\right)\left\{\left[\lambda a+\frac{b \beta}{m}-a(2 p-1) \alpha \tau \frac{4 \mp \sin \theta}{2} V_{e}^{\prime}(\rho)\right] \frac{\Delta^{2}}{2}-c \frac{\beta}{m} \Delta\right\} .
\end{array}\right.
$$

Letting $\rho=\rho_{h}+\hat{\rho}(x, t)$ near the neutral stability condition and introducing the Taylor expansions into the second order of $\hat{\rho}$, we will obtain

$$
\rho V_{e}(\rho) \approx \rho_{h} V_{e}\left(\rho_{h}\right)+\left.\left(\rho V_{e}\right)_{\rho}\right|_{\rho=\rho_{h}} \hat{\rho}+\left.\frac{1}{2}\left(\rho V_{e}\right)_{\rho \rho}\right|_{\rho=\rho_{h}} \hat{\rho}^{2} .
$$

Bringing equation (32) into equation (39), and then turning $\hat{\rho}$ to $\rho$, we can derive that

$$
-c \rho_{z}+\left[\left(\rho V_{e}\right)_{\rho}+\left(\rho V_{e}\right)_{\rho \rho} \rho\right] \rho_{z}+b_{1} \rho_{z z}+b_{2} \rho_{z z z}=0 .
$$

Aiming at obtaining the standard KdV-Burgers equation, we perform the following transformations:

$$
\begin{aligned}
U & =-\left[\left(\rho V_{e}\right)_{\rho}+\left(\rho V_{e}\right)_{\rho \rho} \rho\right], \\
X & =m x, \\
T & =-m t .
\end{aligned}
$$

Putting equation (41) into equation (40), the KdVBurgers equation is received as follows:

$$
U_{T}+U U_{X}-m b_{1} U_{X X}-m^{2} b_{2} U_{X X X}=0 .
$$

One analytic solution of the above KdV-Burgers equation is

$$
U=-\frac{3\left(-m b_{1}\right)^{2}}{25\left(-m^{2} b_{2}\right)}\left[1+2 \tanh \left( \pm \frac{-m b_{1}}{10 m^{2}}\right)\left(X+\frac{6\left(-m b_{1}\right)^{2}}{25\left(-m^{2} b_{2}\right)} T+\zeta_{0}\right)+\tanh ^{2}\left( \pm \frac{-m b_{1}}{10 m^{2}}\right)\left(X+\frac{6\left(-m b_{1}\right)^{2}}{25\left(-m^{2} b_{2}\right)} T+\zeta_{0}\right)\right]
$$

in which $\zeta_{0}$ is an arbitrary constant.

Therefore, for the proposed new macro-hydrodynamic model, the neutral stability conditions are gotten by linear stability analysis, and the $\mathrm{KdV}$-Burgers equation describing traffic congestion is acquired by nonlinear analysis near the neutral stability curve, and the KdVBurgers equation solitary wave solution of vehicle density is obtained. 


\section{Numerical Simulation}

The stability of the model is determined by the slope angle $\theta$ of the road and the sensitivity coefficient $\beta$ of the angle difference of the electronic throttle. In order to facilitate the study of whether the proposed model equations (18) can explain the phase transition phenomenon of traffic flow on the slope road, we discretize the model equation (18) as follows:

$$
\begin{aligned}
v_{x x} & =\frac{v_{i+1}^{j}-2 v_{i}^{j}+v_{i-1}^{j}}{(\Delta x)^{2}}, \\
v_{x t} & =\frac{\left(v_{i+1}^{j+1}-v_{i}^{j+1}\right)-\left(v_{i+1}^{j}-v_{i}^{j}\right)}{\Delta t \times \Delta x}, \\
\rho_{i}^{j+1} & =\rho_{i}^{j}+\frac{\Delta t}{\Delta x} \rho_{i}^{j}\left(v_{i}^{j}-v_{i+1}^{j}\right)+\frac{\Delta t}{\Delta x} v_{i}^{j}\left(\rho_{i-1}^{j}-\rho_{i}^{j}\right) .
\end{aligned}
$$

(a) If the traffic is heavy (i.e., $v_{i}^{j}<c_{i}^{j}$ )

$$
\begin{aligned}
v_{i}^{j+1}= & v_{i}^{j}-\left(v_{i+1}^{j}-v_{i}^{j}\right)\left(v_{i}^{j}-c_{i}^{j}\right) \frac{\Delta t}{\Delta x} \\
& +a\left[\frac{4 \mp \sin \theta}{2} V_{e}\left(\rho_{i}^{j}\right)-v_{i}^{j}\right] \Delta t \\
& +\left(c_{i}^{j}-\frac{\beta}{m} \Delta v_{c}\right) \frac{1}{2} \Delta \frac{v_{i+1}^{j}-2 v_{i}^{j}+v_{i+1}^{j}}{(\Delta x)^{2}} \Delta t \\
& +\frac{\beta}{m} \Delta \frac{v_{i+1}^{j+1}-v_{i}^{j+1}-v_{i+1}^{j}+v_{i}^{j}}{\Delta x} .
\end{aligned}
$$

(b) If the traffic is light (i.e., $v_{i}^{j} \geq c_{i}^{j}$ )

$$
\begin{aligned}
v_{i}^{j+1}= & v_{i}^{j}-\left(v_{i+1}^{j}-v_{i}^{j}\right)\left(v_{i}^{j}-c_{i}^{j}\right) \frac{\Delta t}{\Delta x} \\
& +a\left[\frac{4 \mp \sin \theta}{2} V_{e}\left(\rho_{i}^{j}\right)-v_{i}^{j}\right] \Delta t \\
& +\left(c_{i}^{j}-\frac{\beta}{m} \Delta v_{c}\right) \frac{1}{2} \Delta \frac{v_{i+1}^{j}-2 v_{i}^{j}+v_{i+1}^{j}}{(\Delta x)^{2}} \Delta t \\
& +\frac{\beta}{m} \Delta \frac{v_{i+1}^{j+1}-v_{i}^{j+1}-v_{i+1}^{j}+v_{i}^{j}}{\Delta x},
\end{aligned}
$$

where $c_{i}^{j}=\left[\lambda+b \beta / m-a(2 p-1) \alpha \tau \rho_{i}^{j 2}(4 \mp \sin \theta / 2) V_{e}\left(\rho_{i}^{j}\right)\right] \Delta+$ $(\beta / m) \Delta\left(v_{i+1}^{j}-v_{i}^{j} / \Delta x\right), \rho_{i}^{j}$ and $v_{i}^{j}$ represent the density and the speed under the condition of $(i, j)$, and $j$ and $i$ represent the time and the space section, respectively. Here, the space step and the time step are represented by $\Delta x$ and $\Delta t$.

5.1. Shock Waves and Rarefaction Waves. Firstly, the influence of the proposed model on the spatial and temporal evolution of density and velocity under congestion and rarefaction conditions after adding small perturbations is studied, which corresponds to two Riemann initial conditions, written as follows:

$$
\begin{gathered}
\rho_{\text {up }}^{1}=0.04(\mathrm{veh} / \mathrm{m}), \\
\rho_{\text {down }}^{1}=0.18(\mathrm{veh} / \mathrm{m}), \\
\rho_{\text {up }}^{2}=0.18(\mathrm{veh} / \mathrm{m}), \\
\rho_{\text {down }}^{2}=0.04(\mathrm{veh} / \mathrm{m}),
\end{gathered}
$$

among them, $\rho_{\text {up }}^{1}$ and $\rho_{\text {up }}^{2}$ are the density of upstream vehicles and $\rho_{\text {down }}^{1}$ and $\rho_{\text {down }}^{2}$ are the density of downstream vehicles. Equation (47a) is the traffic flow from smooth to congested, and the traffic density wave shows shock wave propagation. Equation (47b) is the traffic density wave that propagates in rarefaction wave when the congested traffic flow is dispersed. The initial conditions of velocity corresponding to the initial conditions of Riemann are as follows:

$$
\begin{gathered}
v_{\text {up }}^{1,2}=v_{e}\left(\rho_{\text {up }}^{1,2}\right), \\
v_{\text {down }}^{1,2}=v_{e}\left(\rho_{\text {down }}^{1,2}\right) .
\end{gathered}
$$

The result by comparing equation (18), the balanced velocity function is equations (45) and (46):

$$
v_{e}=v_{f}\left[1-\exp \left(1-\exp \left(\frac{n_{m}}{v_{f}}\left(\frac{\rho_{m}}{\rho}-1\right)\right)\right)\right],
$$

among them, $v_{f}$ is the free flow speed, $\rho_{m}$ is the vehicle density when the road is congested, and $n_{m}$ is the propagation speed of the density wave in the congested state. The total length of road $20 \mathrm{~km}$ is divided into 200 equal length units; each time step $\Delta t$ is $1 s$, using the following other parameters: $a=0.4(1 / \mathrm{s}), \lambda=0.5, \Delta=10 \mathrm{~m}, \quad \tau=0.5 \mathrm{~s}$, $\alpha=0.8, m=5, b=7, \beta=1.2, \theta=4$. The traffic flow of the new model proposed by the numerical simulation changes with time under two initial Riemann conditions, as shown in Figures 3 to 6 .

In the macro traffic flow, the shock wave refers to the stable traffic flow with low density. Because of the sudden disturbance, the density of the traffic flow at a certain location increases rapidly, and the change of density is close to the step function. In the actual traffic scene, the shock wave is often generated in traffic flow. For example, when the number of lanes or the available area of the road is reduced, the traffic flow in front of the road will form a shock wave, and where there is a disturbance, the traffic flow will suddenly increase. For the stable traffic flow, the traffic density wave generated by the sudden disturbance that makes the traffic flow density increase sharply is the shock wave.

The sparse wave in the macroscopic traffic flow refers to the stable traffic flow with a large initial density. At a certain position and time, because the density of the external disturbance traffic flow suddenly decreases, the traffic flow wave generated in the traffic flow is called the sparse wave. For example, in actual traffic, when a traffic flow with high density stops at a red light and waits, the waiting vehicle pulls out after the red light turns green, the density 


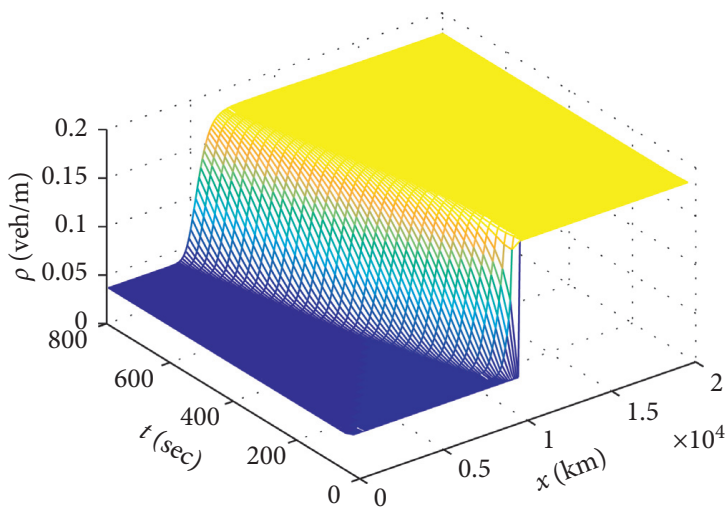

(a)

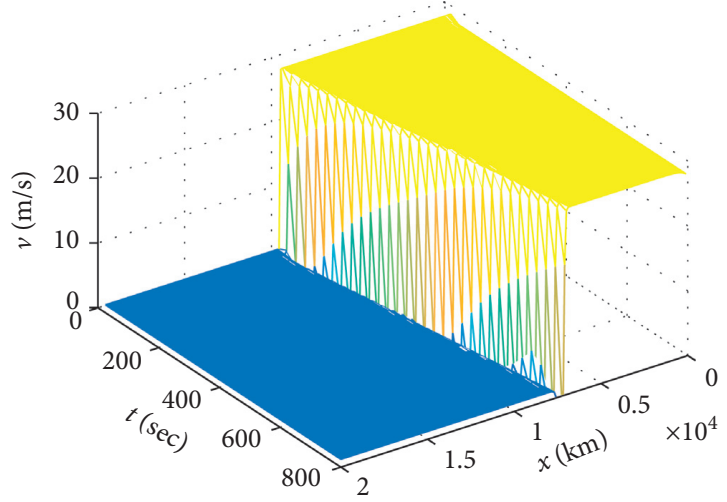

(c)

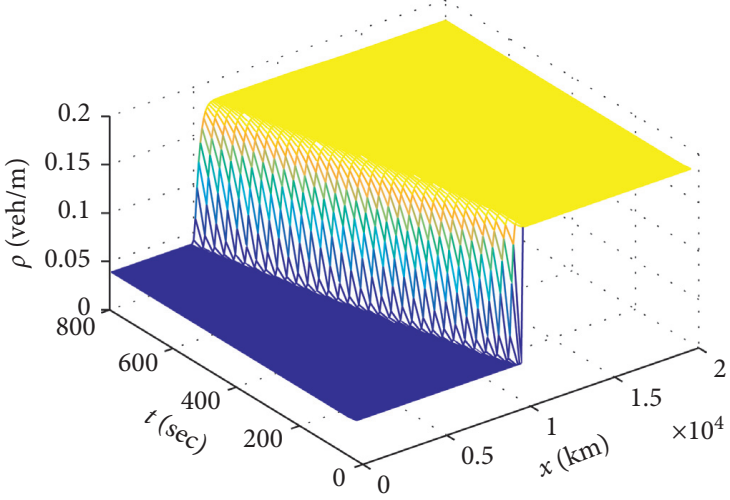

(e)

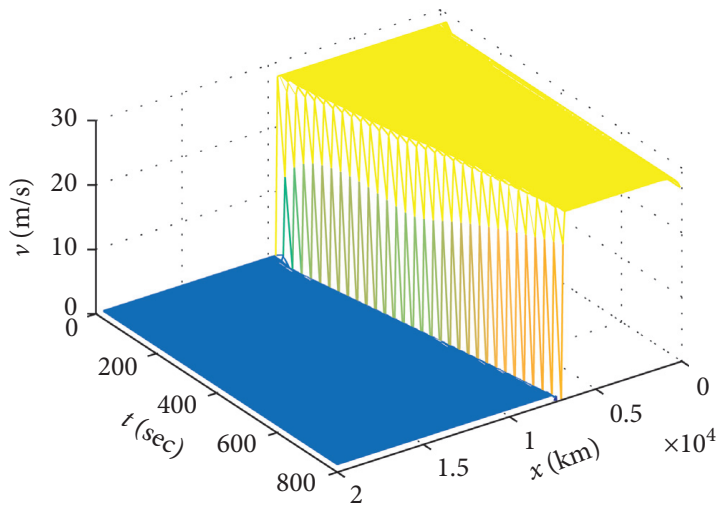

(g)

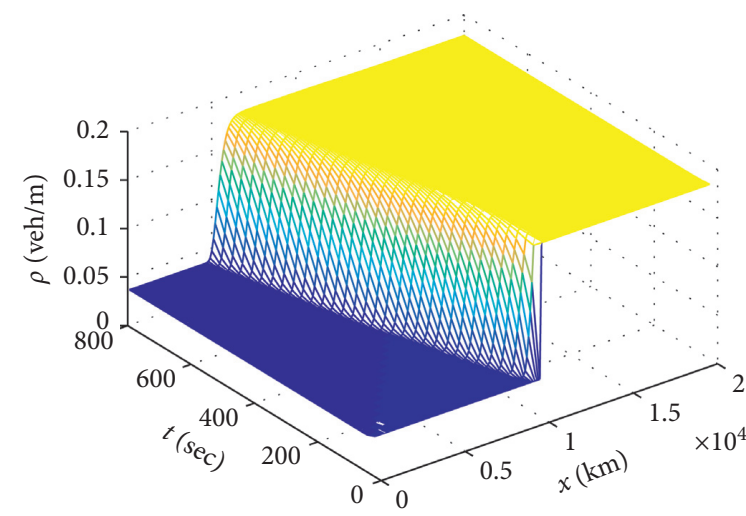

(b)

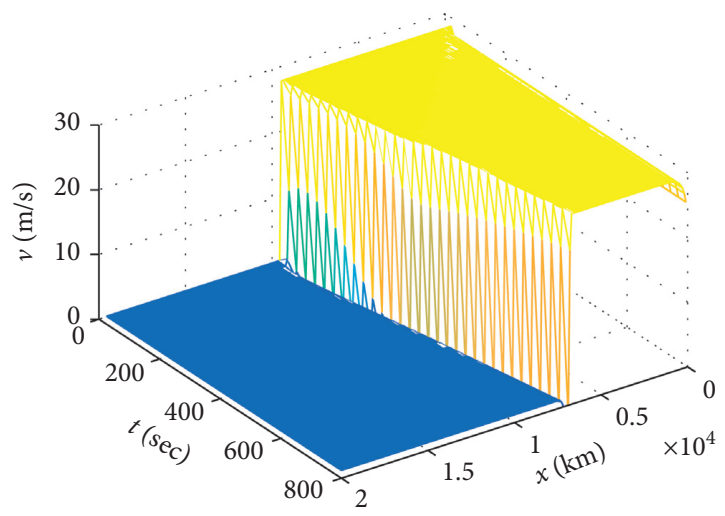

(d)

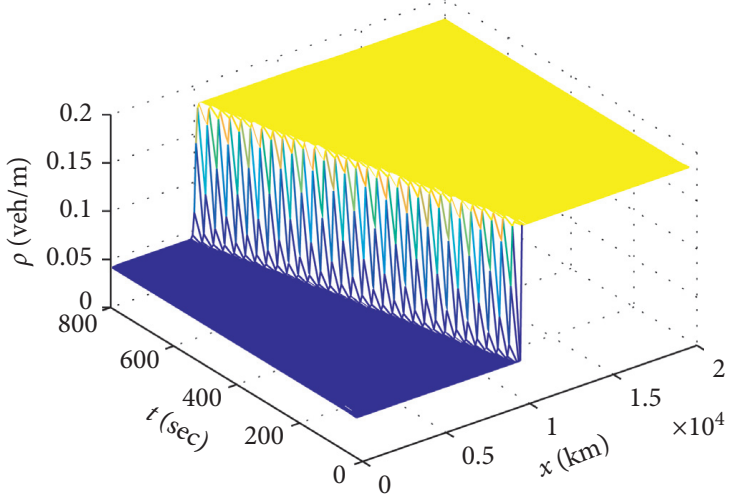

(f)

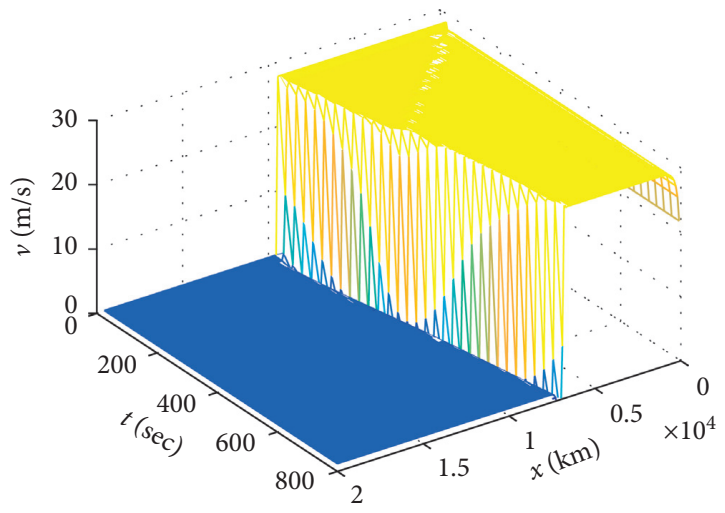

(h)

Figure 3: The shock wave in the initial Riemann condition (47a) on uphill slope. (a) Aggressive driving with ET. (b) Timid driving with ET. (c) Aggressive driving with ET. (d) Timid driving with ET. (e) Aggressive driving without ET. (f) Timid driving without ET. (g) Aggressive driving without ET. (h) Timid driving without ET. 


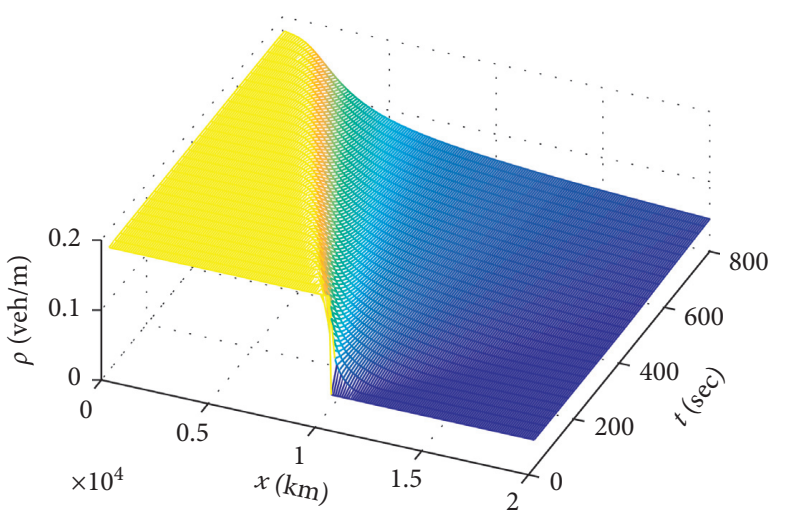

(a)

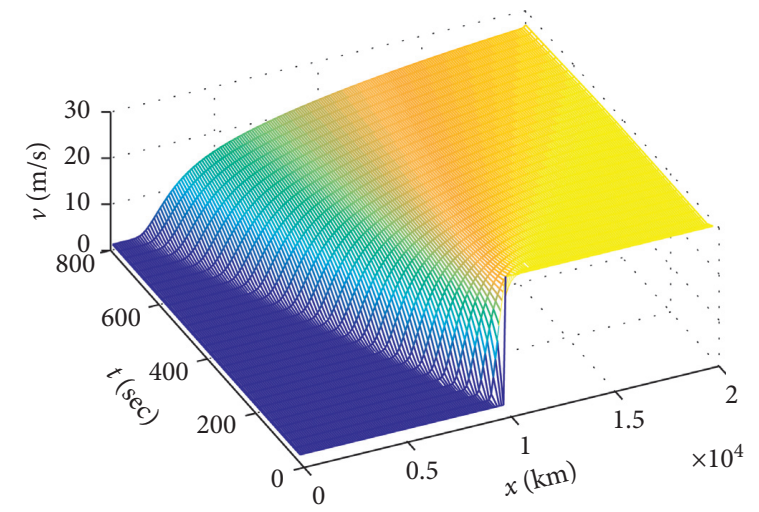

(c)

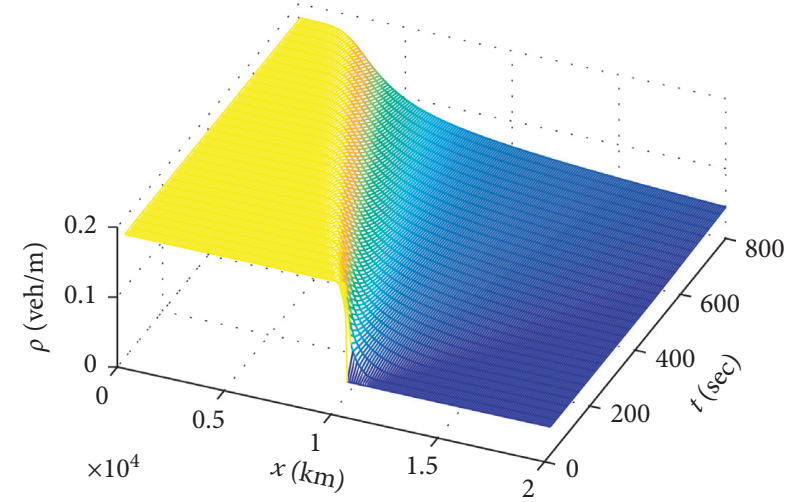

(e)

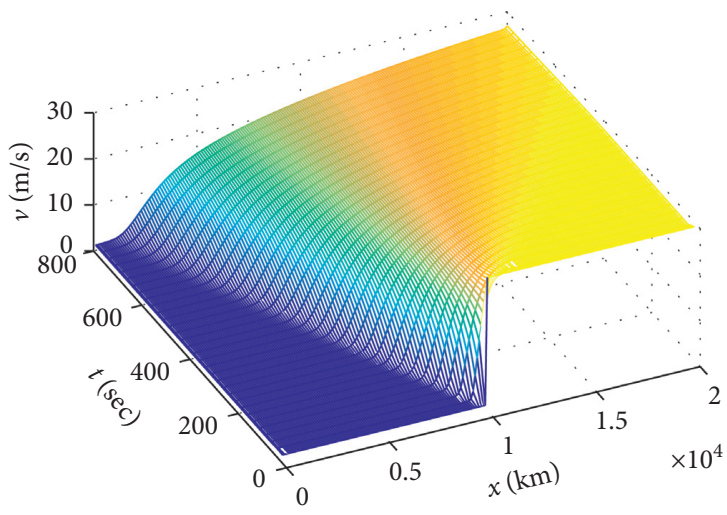

(g)

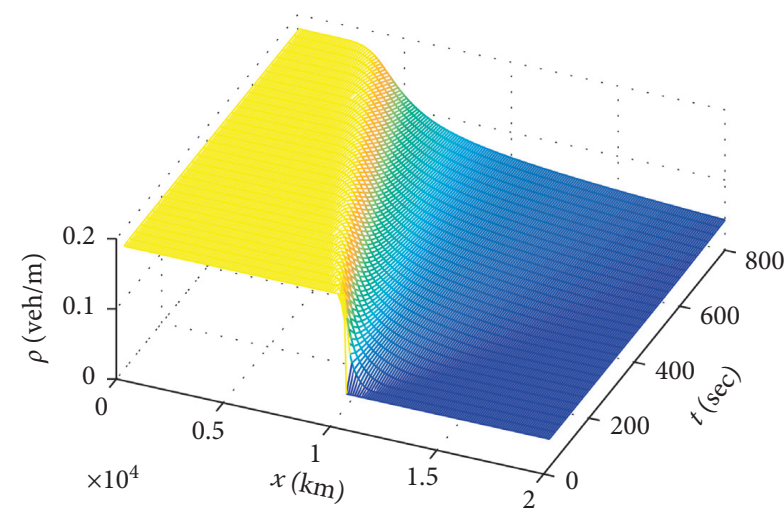

(b)

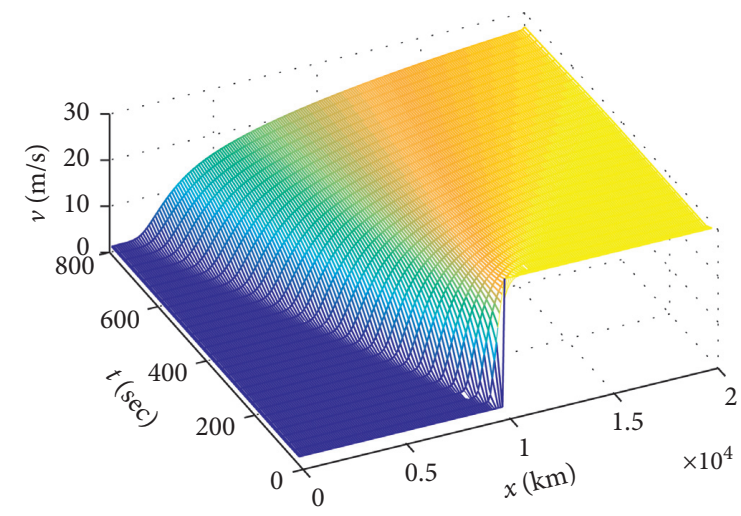

(d)

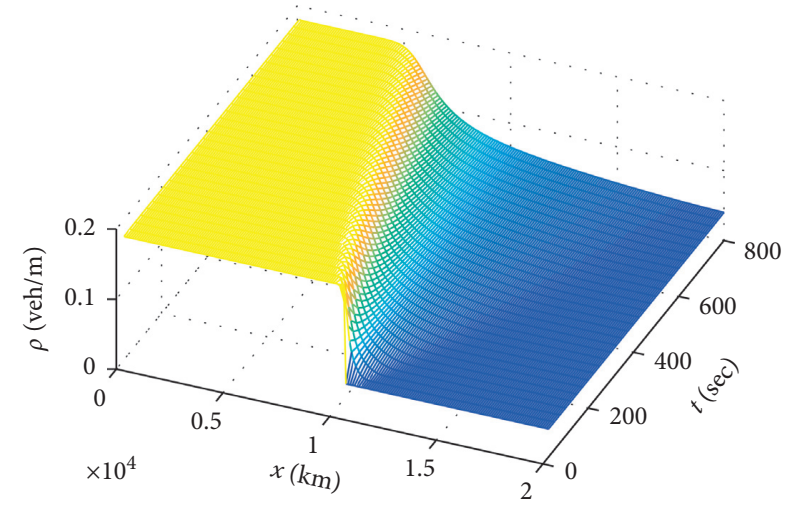

(f)

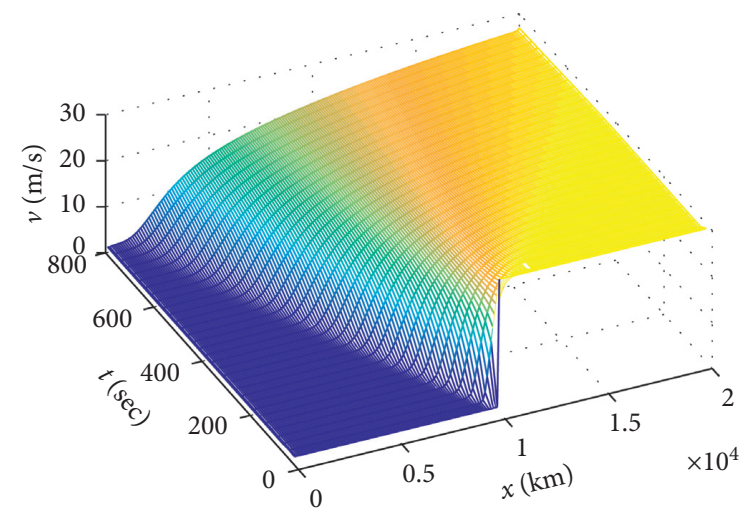

(h)

FIgURE 4: The rarefaction wave in the initial Riemann condition (47b) on uphill slope. (a) Aggressive driving with ET. (b) Timid driving with ET. (c) Aggressive driving with ET. (d) Timid driving with ET. (e) Aggressive driving without ET. (f) Timid driving without ET. (g) Aggressive driving without ET. (h) Timid driving without ET. 


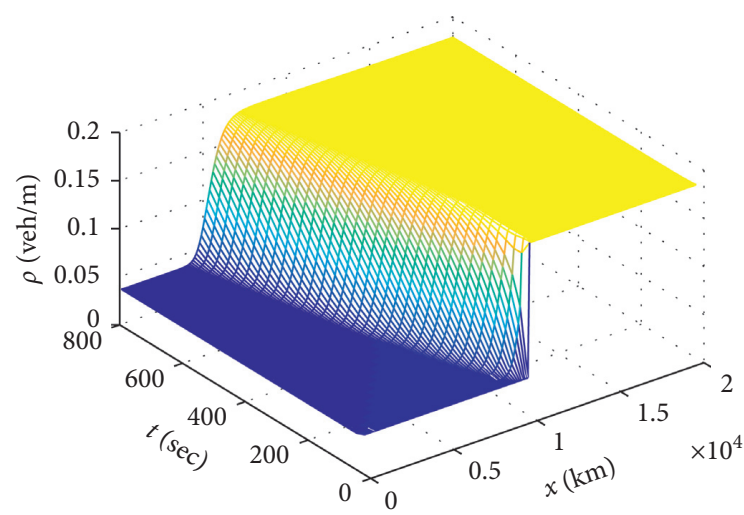

(a)

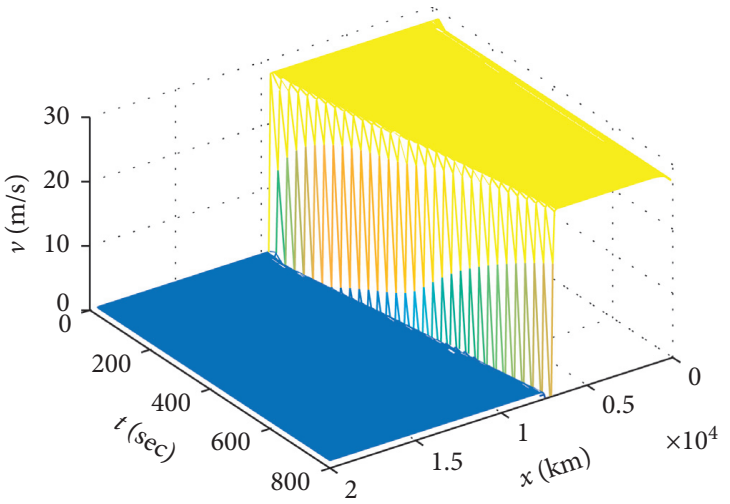

(c)

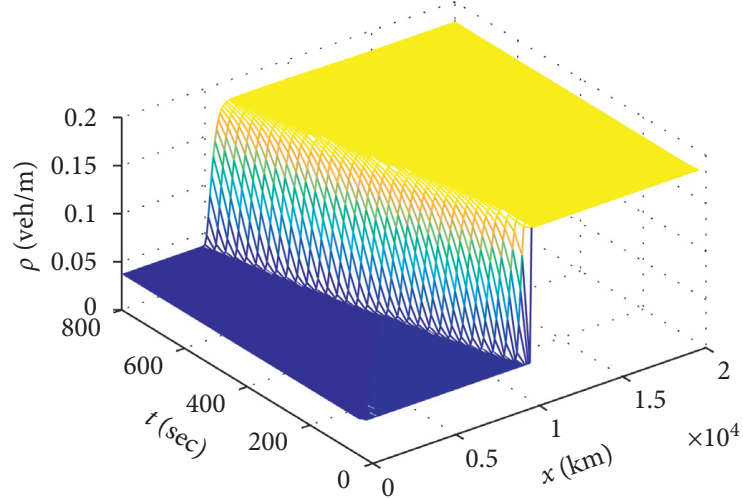

(e)

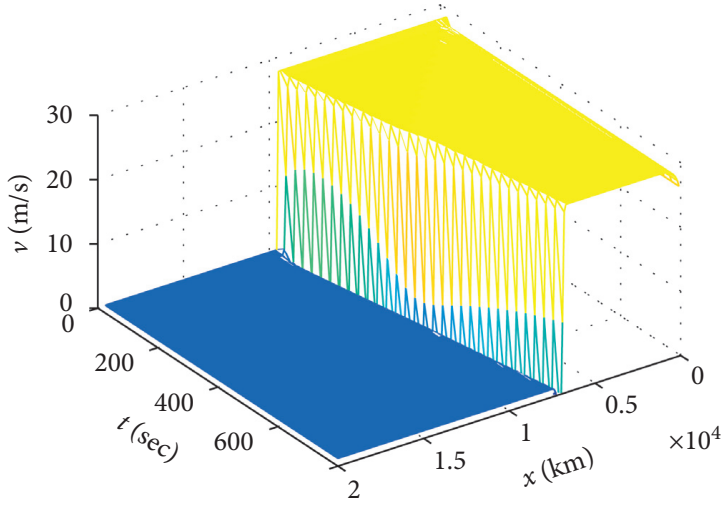

(g)

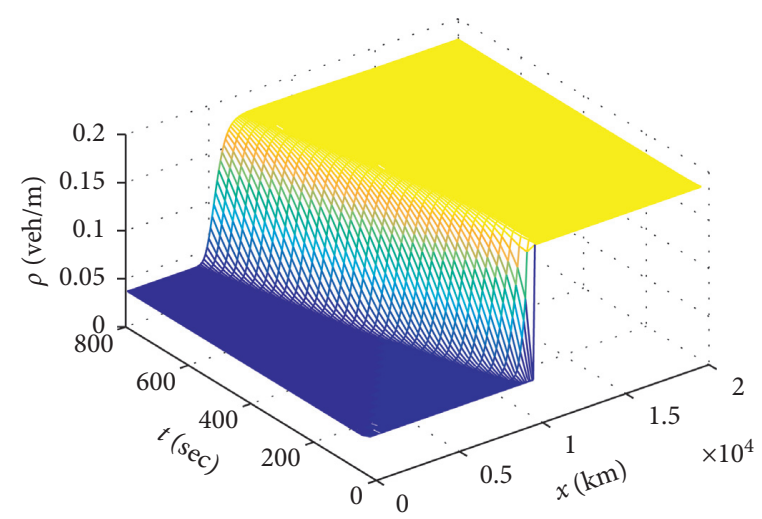

(b)

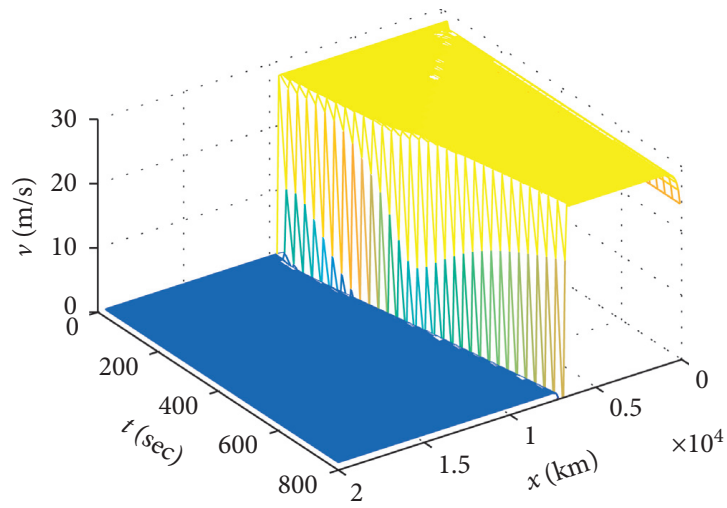

(d)

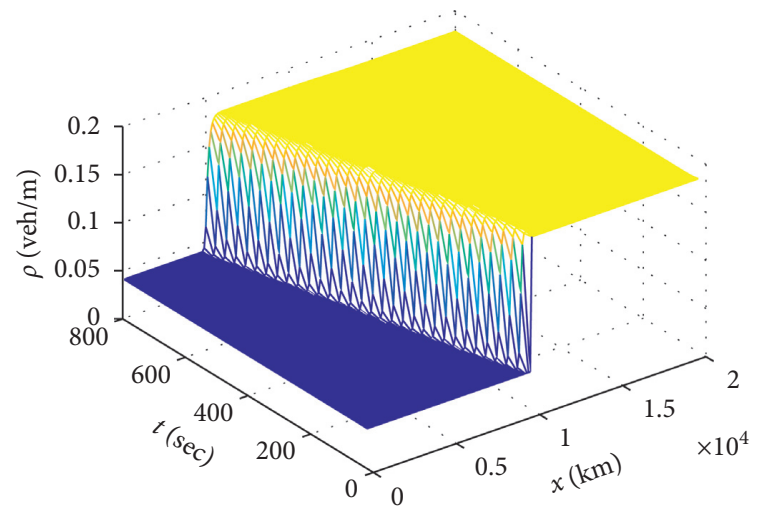

(f)

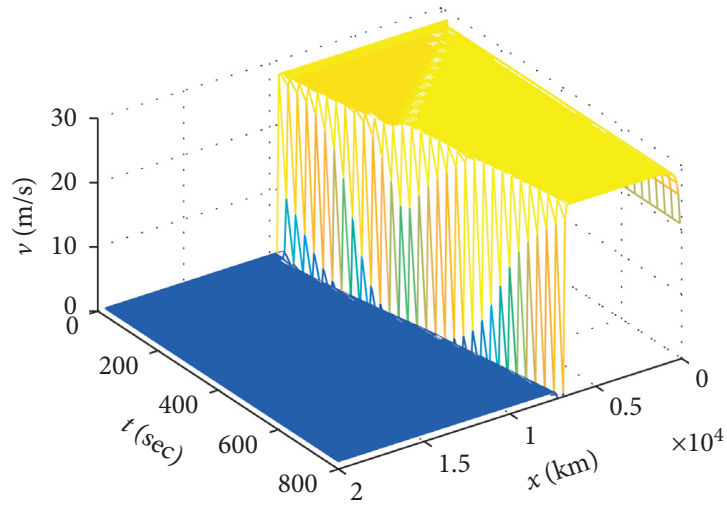

(h)

Figure 5: The shock wave in the initial Riemann condition (47a) on downhill slope. (a) Aggressive driving with ET. (b) Timid driving with ET. (c) Aggressive driving with ET. (d) Timid driving with ET. (e) Aggressive driving without ET. (f) Timid driving without ET. (g) Aggressive driving without ET. (h) Timid driving without ET. 


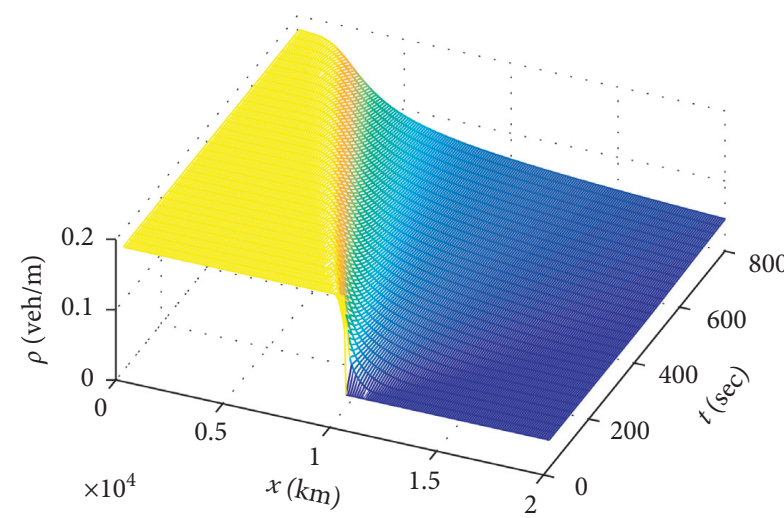

(a)

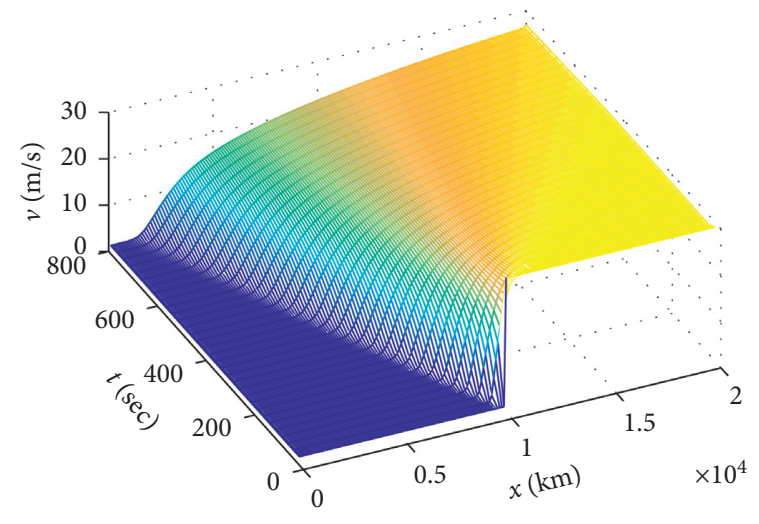

(c)

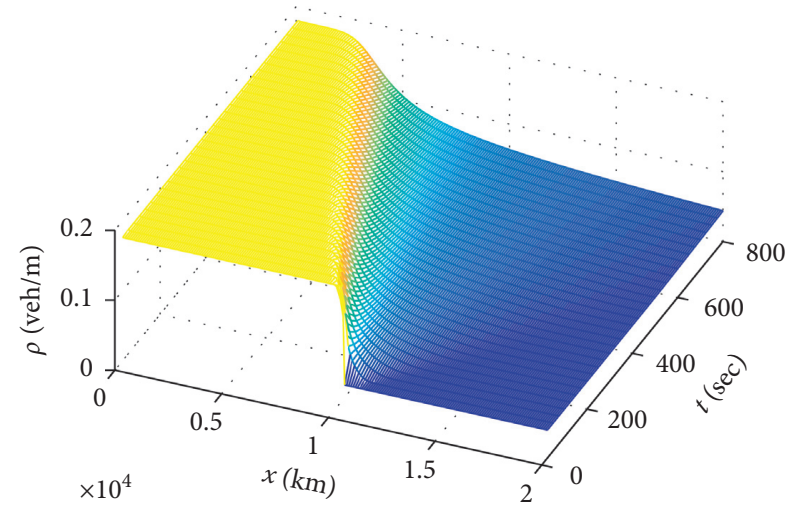

(e)

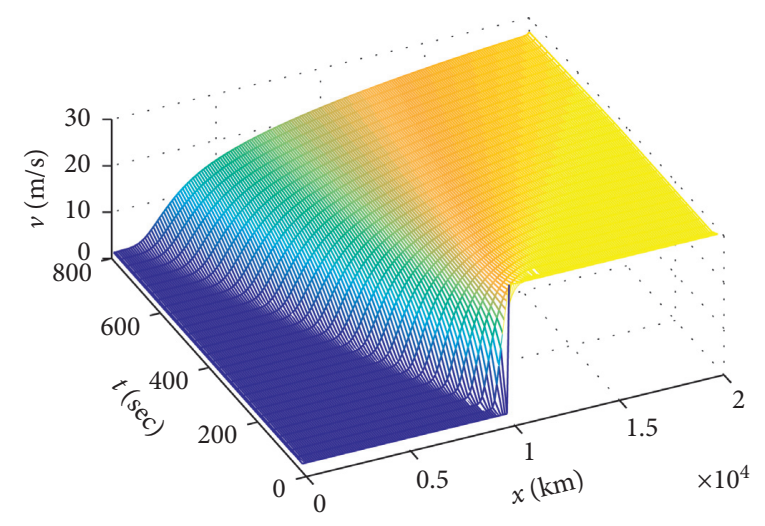

(g)

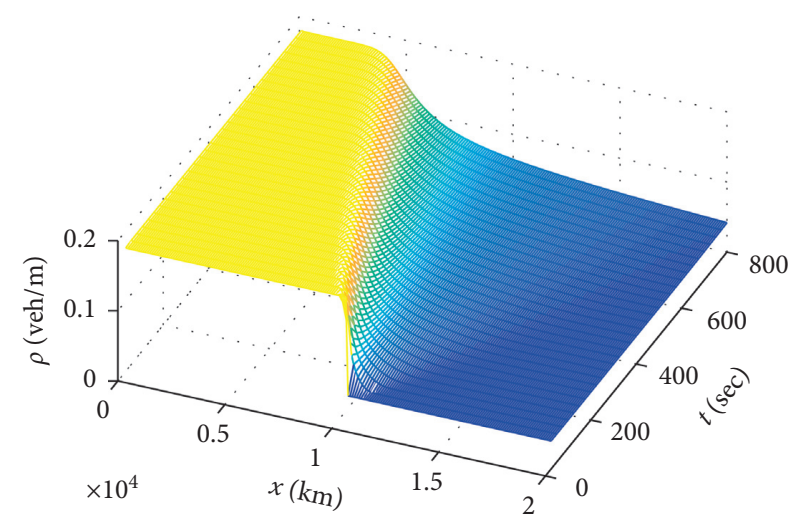

(b)

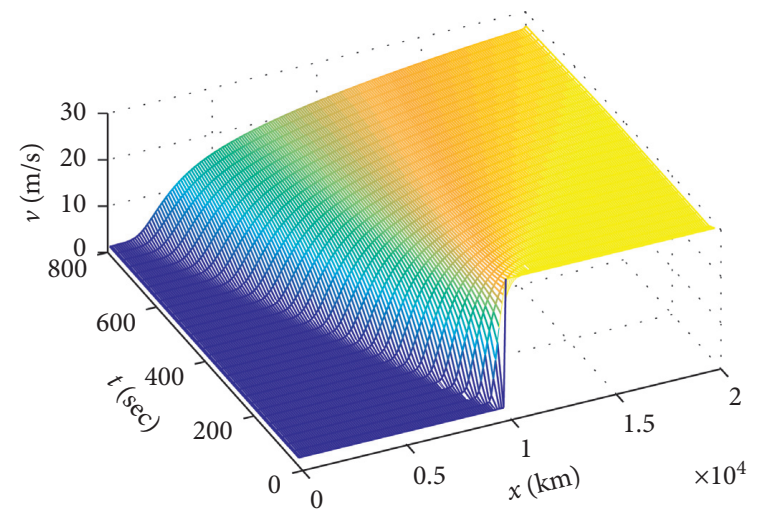

(d)

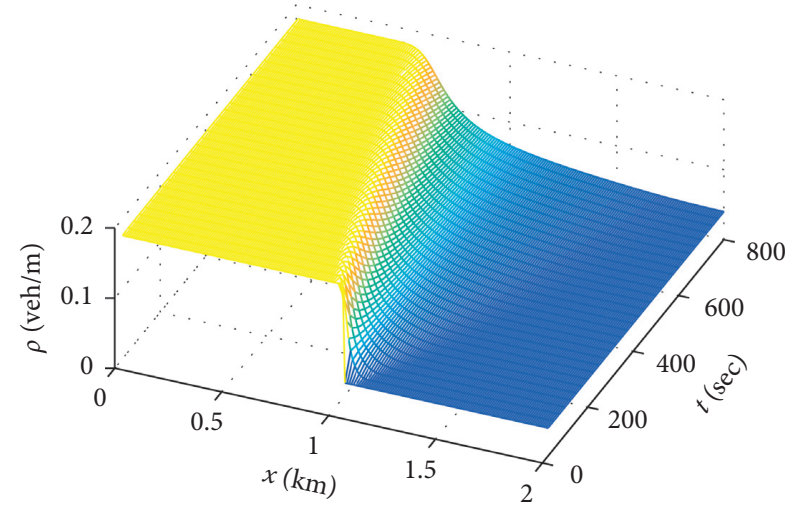

(f)

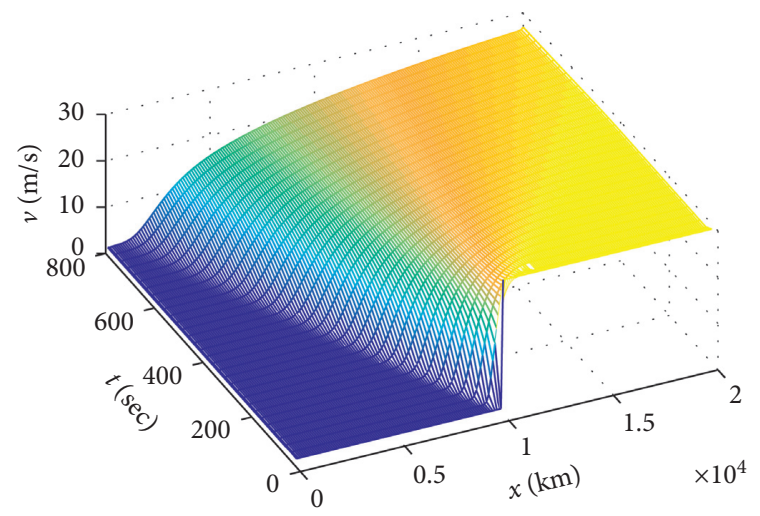

(h)

Figure 6: The rarefaction wave in the initial Riemann condition (47b) on downhill slope. (a) Aggressive driving with ET. (b) Timid driving with ET. (c) Aggressive driving with ET. (d) Timid driving with ET. (e) Aggressive driving without ET. (f) Timid driving without ET. (g) Aggressive driving without ET. (h) Timid driving without ET. 
decreases, and the resulting traffic flow fluctuations become sparse waves.

As shown in Figures 3 and 4, the effects of driver's driving characteristics and electronic throttle angle are brought into the uphill road with a slope angle of $\theta=4$. Figure 3 reflects the evolution of density and velocity of traffic flow during the formation of shock wave in traffic congestion. Figure 4 shows the variation of density and velocity of traffic flow density wave with time in the process of rarefaction wave formation. Figures 3(a) and 3(c) and Figures 4(a) and 4(c) are the density evolution of radical driving. Figures 3(b) and 3(d) and Figures 4(b) and 4(d) are the density evolution of timid driving. The right side is the corresponding evolution chart without considering the influence of electronic throttle angle. Figure 3 shows the reverse propagation of shock wave. In Figure 4, we can see that the moving front of sparse wave moves steadily with time.

In the process of traffic flow from unblocked to congested, because the upstream traffic is unblocked at the beginning and the downstream traffic is blocked, when the upstream traffic flow encounters the downstream congestion, the upstream traffic slows down one after another, which leads to the change of traffic flow from free flow to blocked flow. When all roads are completely blocked, all vehicles almost stop driving and turn into blocked flow. The reverse propagation of shock wave is well demonstrated.

On the contrary, in the process of traffic congestion dissipation, we can see from the figure that because the new model is anisotropic traffic flow model, the speed of traffic flow is increasing, the number of vehicles in unit length is decreasing, and the density of traffic flow is decreasing. This is because the upstream traffic is blocked at the beginning, and the downstream traffic is unblocked. Upstream vehicles can accelerate to leave the blocked area. It can be seen that the waves front of sparse waves will move smoothly with the passage of time. Obviously, under different initial Riemann conditions, different frontal motion phenomena will occur between traffic congestion and free traffic flow. At the same time, displacement of shock wave and sparse wave can be observed.

It can be concluded that radical driving can make shock wave and sparse wave smoother than timid driving, which is consistent with theoretical analysis. Moreover, the impact of driver's radical driving and electronic throttle angle has a positive impact on both traffic waves, mainly for shock wave. In addition, the impact of radical driving and electronic throttle angle can be obvious to help alleviate traffic congestion. That is to say, the new continuum model can smooth the wave front.

As shown in Figures 5 and 6, the effects of driver's driving characteristics and electronic throttle angle are considered on the downhill road with a slope of 4 . Figure 5 reflects the evolution of density and velocity of traffic flow during the formation of shock wave in traffic congestion. Figure 6 shows the variation of density and velocity of traffic flow density wave with time during the formation of sparse wave. Figures 5(a) and 5(c) and Figures 6(a) and 6(c) are the density evolution of radical driving. Figures 5(b) and 5(d) and Figures 6(b) and 6(d) are the density evolution of timid driving, and the right side is the corresponding evolution chart without considering the influence of electronic throttle angle. Figure 5 shows the reverse propagation of shock wave. In Figure 6, we can see that the moving front of sparse wave moves steadily with time.

Similarly, it can be concluded that, during downhill driving, radical driving can make shock wave and sparse wave smoother than timid driving, which is consistent with theoretical analysis. The impact of driver's radical driving and electronic throttle angle on both traffic waves is a positive impact, which mainly manifests as shock wave. In addition, the impact of radical driving and electronic throttle angle can be clearly obtained to help alleviate traffic congestion. That is to say, the new continuum model can smooth the wave front.

5.2. Slope Angle Parameter $\theta$. In order to observe the evolution of traffic flow density wave more intuitively, the average density $\rho_{0}$ at initial change refers to Herrmann and Kerner [59]:

$$
\rho(x, 0)=\rho_{0}+\Delta \rho_{0}\left\{\cosh ^{-2}\left[\frac{160}{L}\left(x-\frac{5 L}{16}\right)\right]-\frac{1}{4} \cosh ^{-2}\left[\frac{40}{L}\left(x-\frac{11 L}{32}\right)\right]\right\} .
$$

The road length is $L=32.2 \mathrm{~km}$ and the initial density is $\rho_{0}$. In this section, the following periodic boundary conditions are used:

$$
\begin{aligned}
& \rho(L, t)=\rho(0, t), \\
& v(L, t)=v(0, t) .
\end{aligned}
$$

Referring to Kerner and Konhauser [60], we introduce the following expression of the relationship between equilibrium velocity and density as follows:

$$
V_{e}(\rho)=V_{f}\left[\frac{1}{\left(1+\exp \left(\rho / \rho_{m}-0.25 / 0.06\right)\right)}-3.72 \times 10^{-6}\right] \text {, }
$$

where $v_{f}$ and $\rho_{m}$ represent free flow velocity and maximum density, respectively, and the other remaining parameters are set as follows:

$$
\begin{aligned}
v_{f} & =30 \mathrm{~m} / \mathrm{s}, \\
\rho_{m} & =0.2 \mathrm{veh} / \mathrm{m}, \\
\Delta x & =100 \mathrm{~m}, \\
m & =5, \\
b & =7, \\
\beta & =1.2 .
\end{aligned}
$$

Assume that the initial traffic flow is in local equilibrium everywhere; that is, $v(x, 0)=V_{e}(\rho(x, 0))$. On this basis, other parameters are set as follows: $\rho_{m}=0.2 \mathrm{veh} / \mathrm{m}$, $\Delta t=0.5 \mathrm{~s}, a=0.2, \alpha^{\prime}=0.9, \alpha^{\prime \prime}=0.3, \lambda=0.8, \Delta=4$. Considering the driver's driving characteristics on different slope $\theta=0,2,4,6$ and the influence of electronic throttle angle on traffic flow stability, the new extended model is compared 


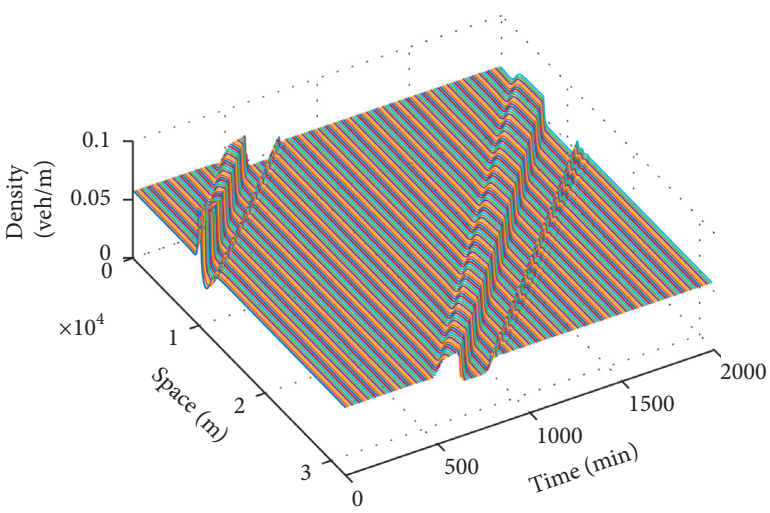

(a)

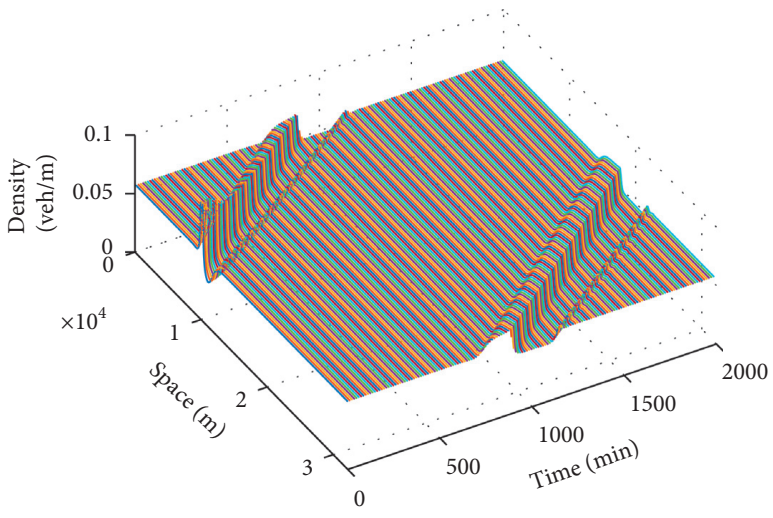

(c)

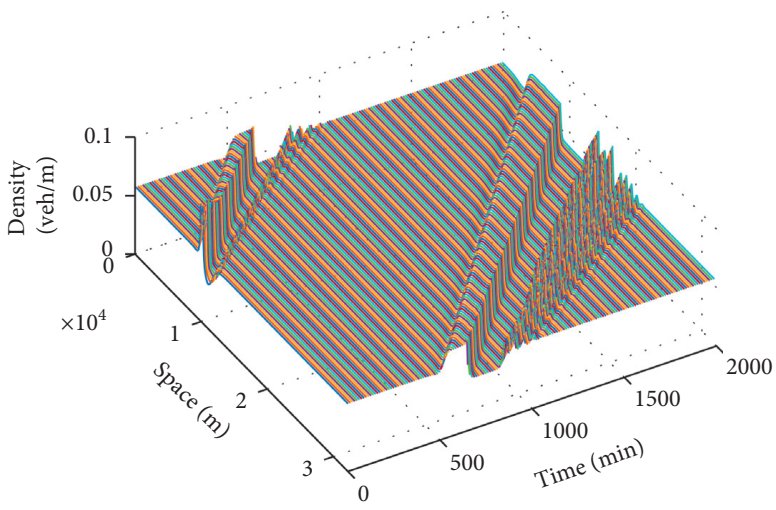

(e)

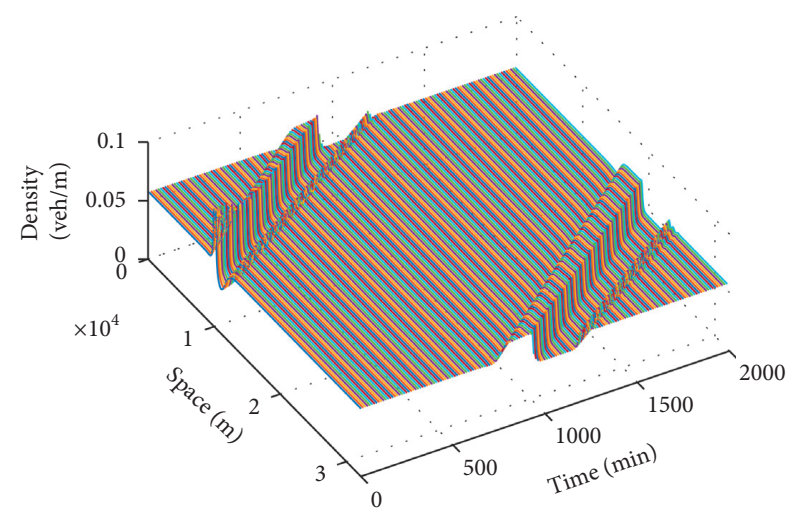

(g)

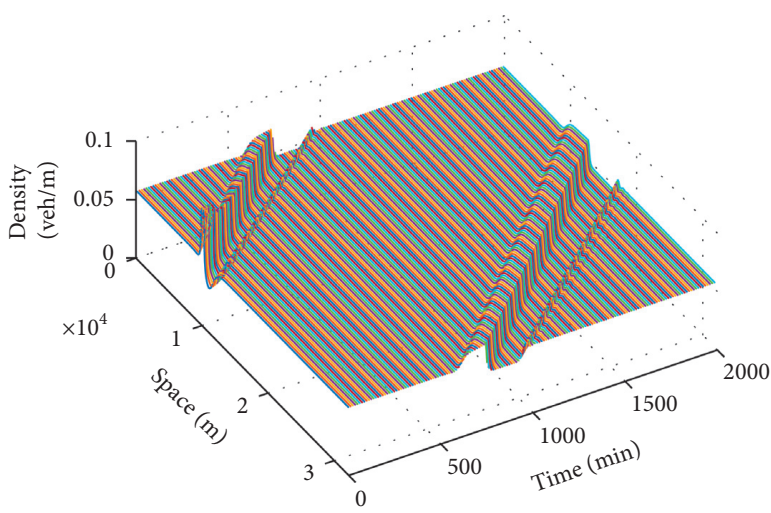

(b)

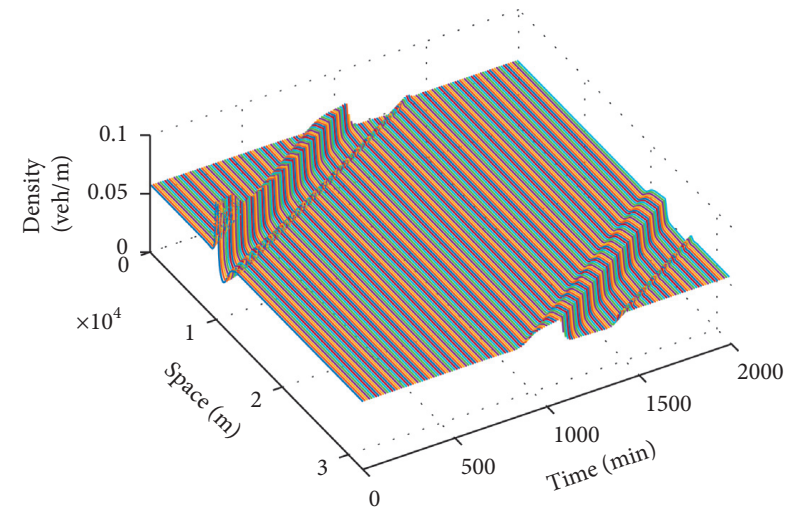

(d)

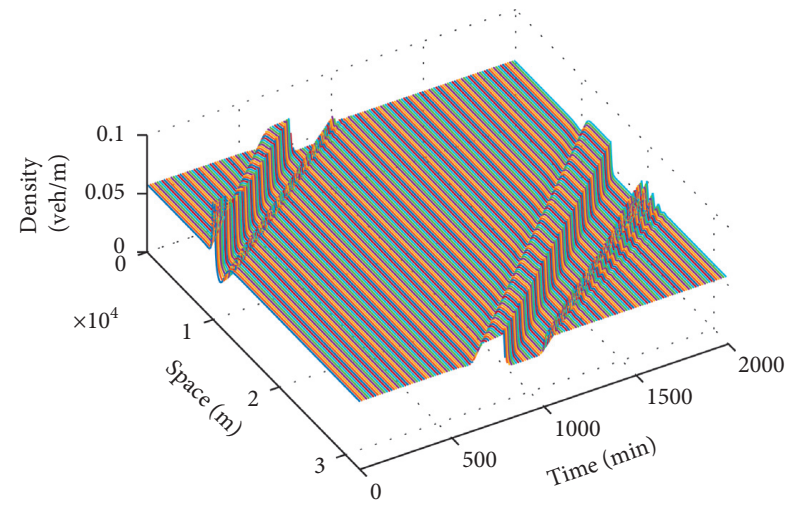

(f)

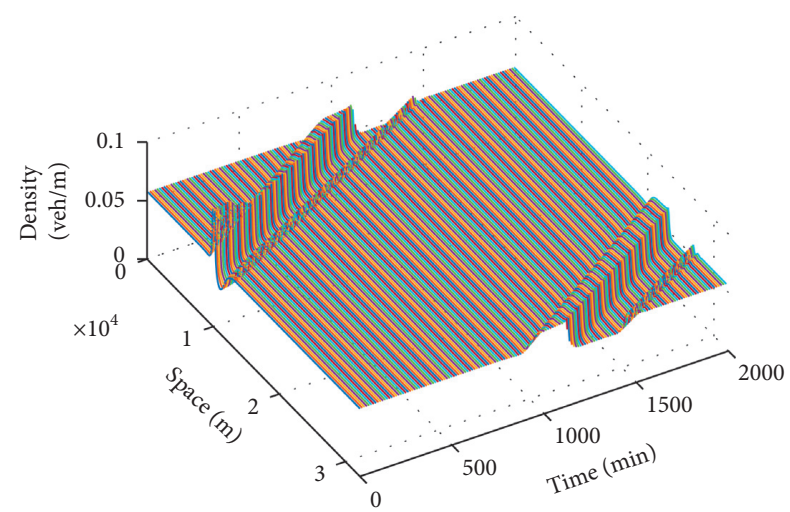

(h)

Figure 7: The evolution of traffic flow density wave in temporal and spatial during driver's aggressive driving uphill. (a) With ET when $\theta=0$. (b) With ET when $\theta=2$. (c) With ET when $\theta=4$. (d) With ET when $\theta=6$. (e) Without ET when $\theta=0$. (f) Without ET when $\theta=2$. (g) Without ET when $\theta=4$. (h) Without ET when $\theta=6$. 


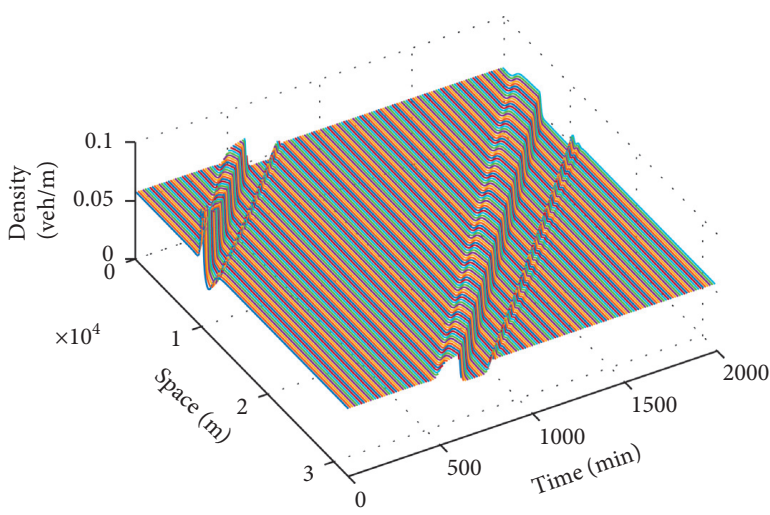

(a)

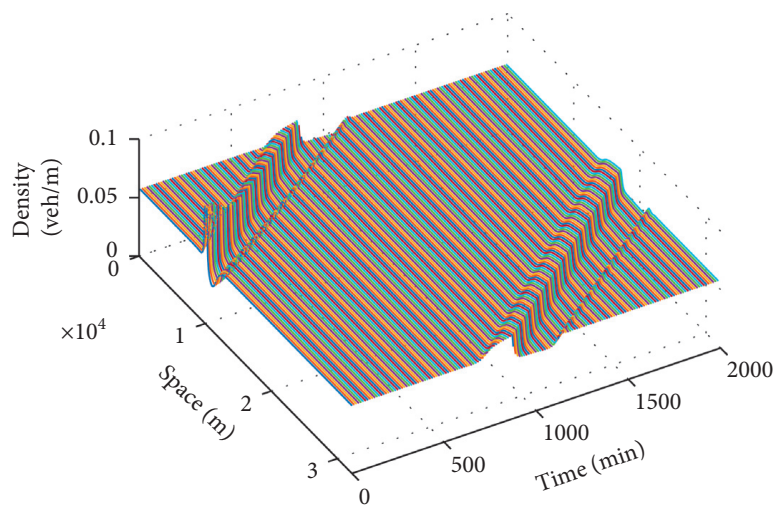

(c)

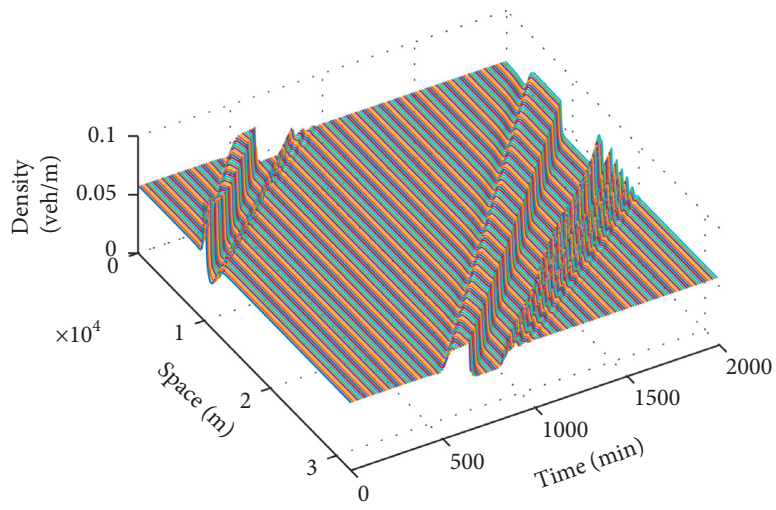

(e)

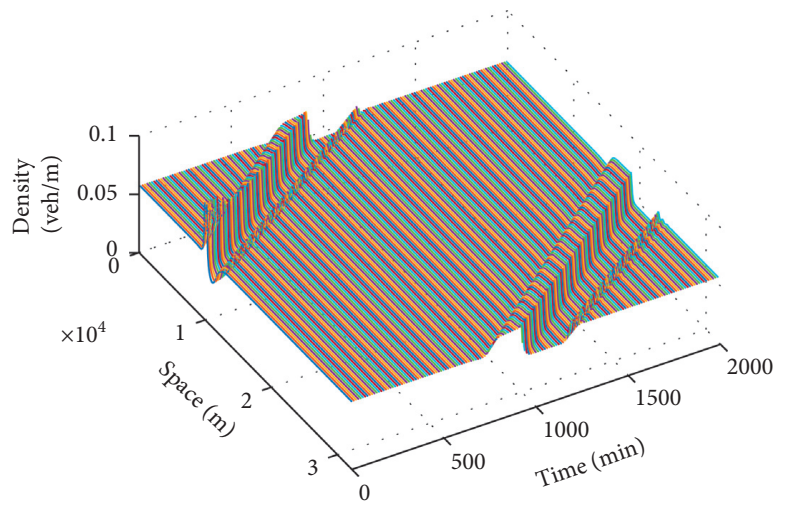

(g)

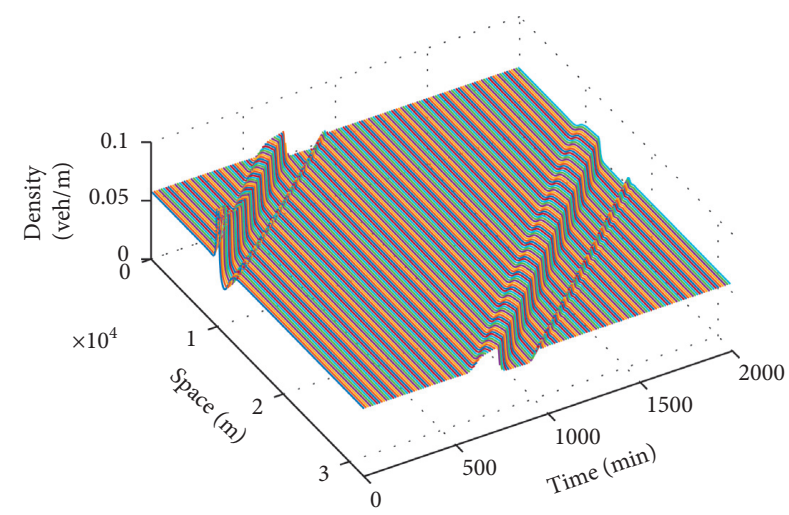

(b)

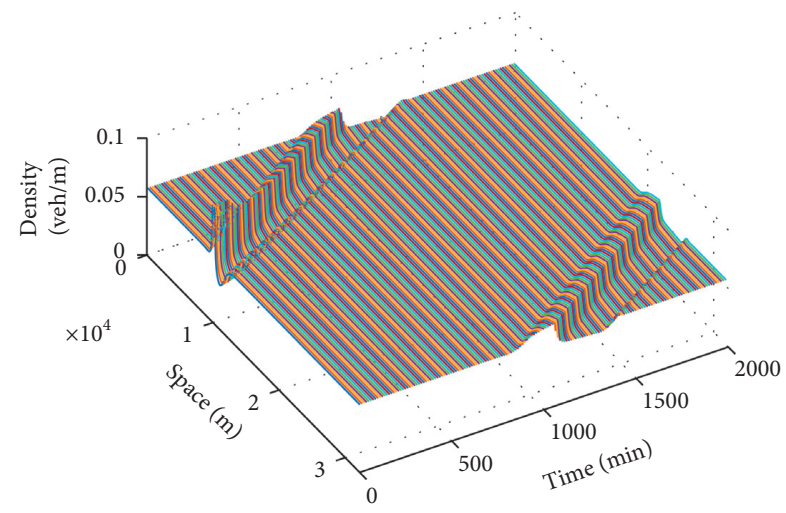

(d)

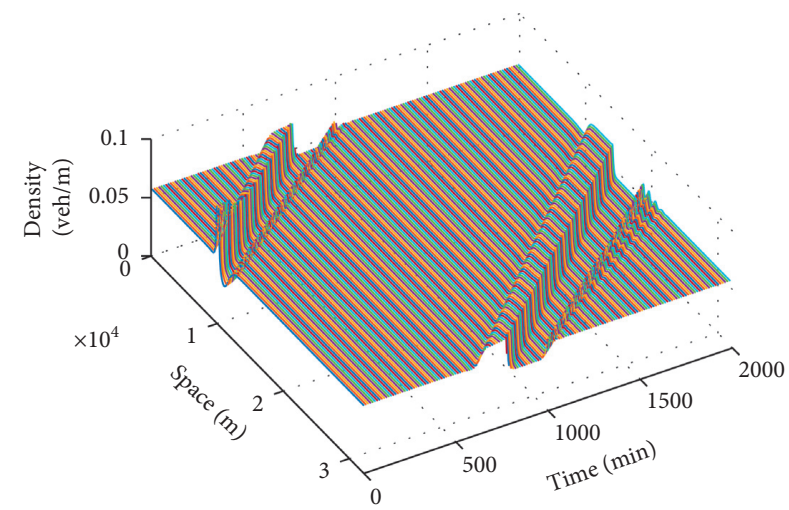

(f)

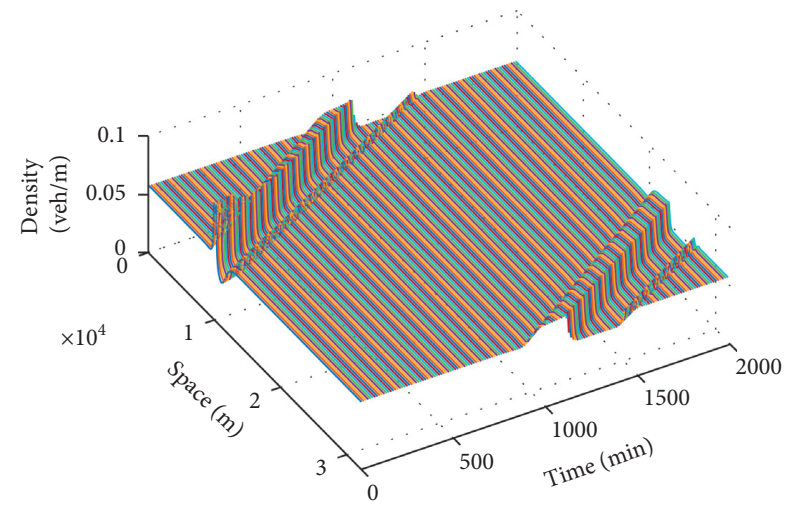

(h)

FIGURE 8: The evolution of traffic flow density wave in temporal and spatial during driver's timid driving uphill. (a) With ET when $\theta=0$. (b) With ET when $\theta=2$. (c) With ET when $\theta=4$. (d) With ET when $\theta=6$. (e) Without ET when $\theta=0$. (f) Without ET when $\theta=2$. (g) Without ET when $\theta=4$. (h) Without ET when $\theta=6$. 


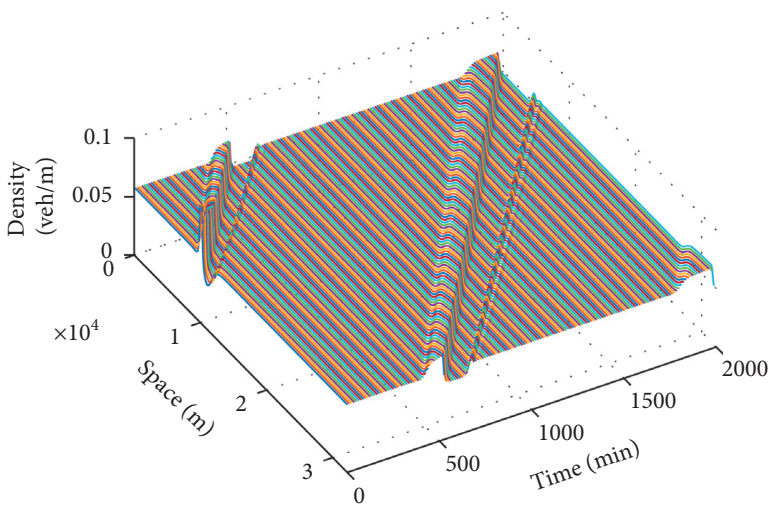

(a)

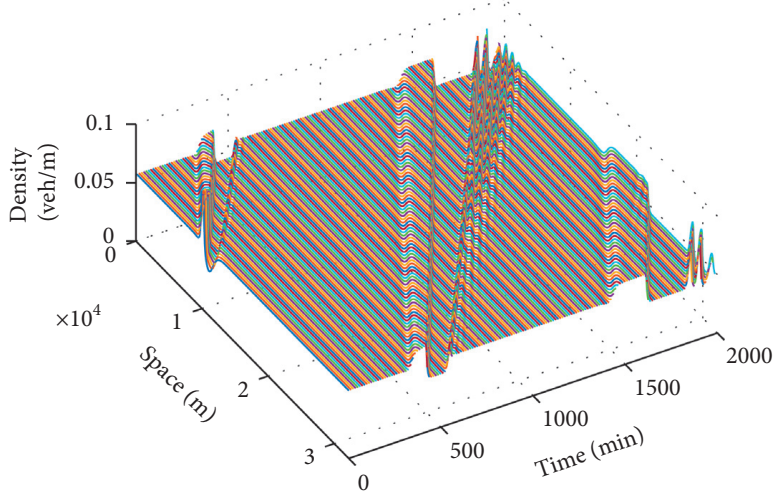

(c)

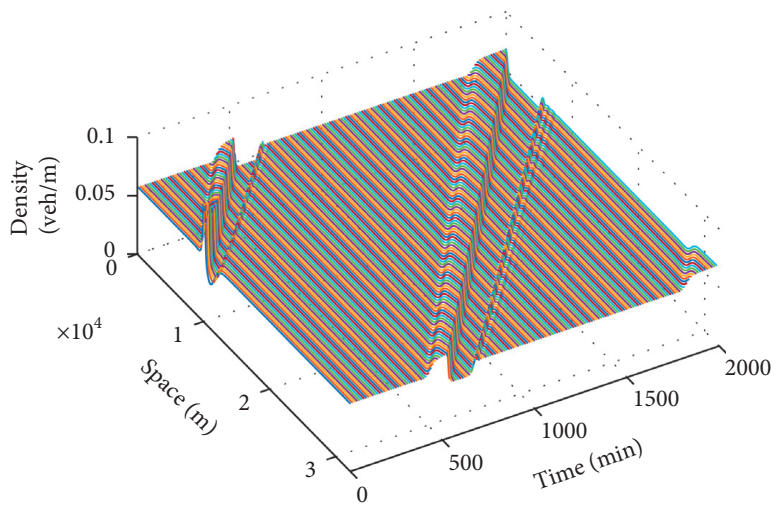

(e)

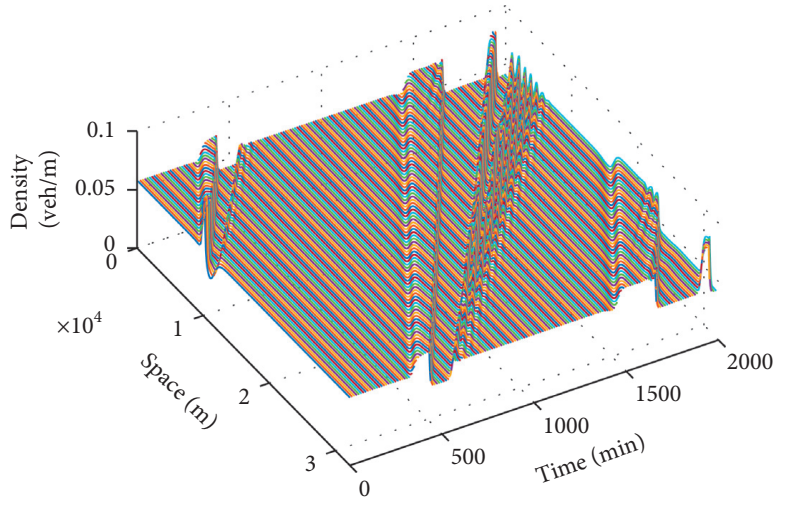

(g)

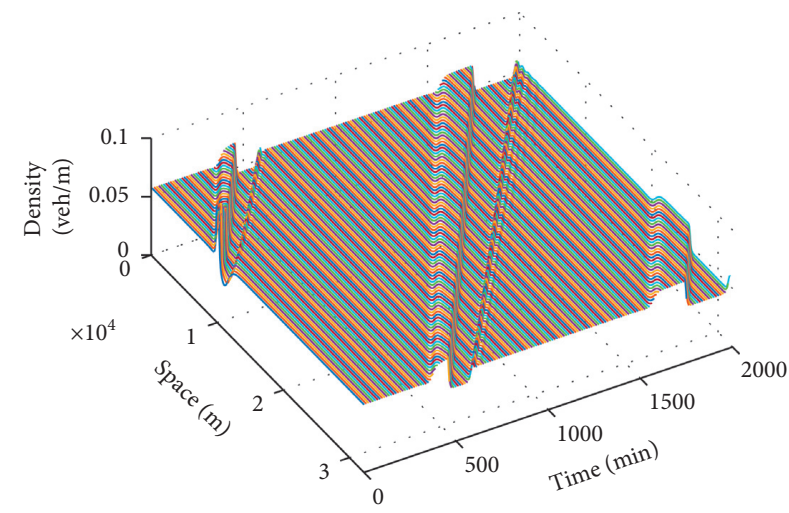

(b)

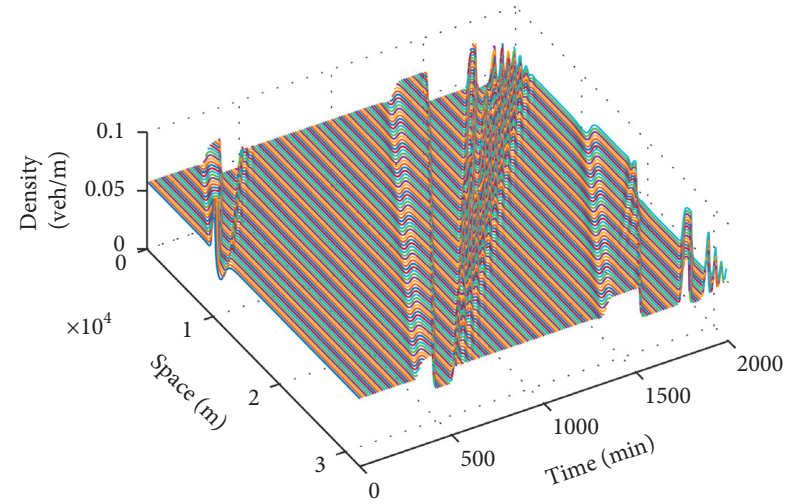

(d)

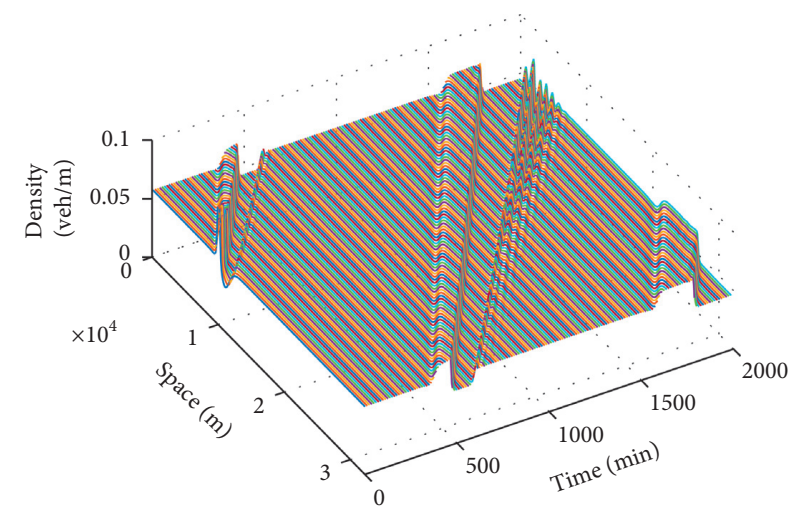

(f)

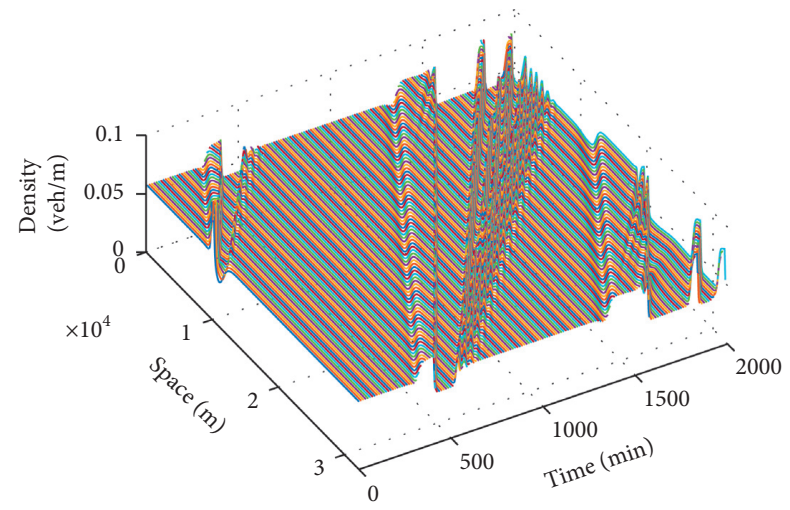

(h)

FIgURE 9: The evolution of traffic flow density wave in temporal and spatial during driver's aggressive driving downhill. (a) With ET when $\theta=0$. (b) With ET when $\theta=2$. (c) With ET when $\theta=4$. (d) With ET when $\theta=6$. (e) Without ET when $\theta=0$. (f) Without ET when $\theta=2$. (g) Without ET when $\theta=4$. (h) Without ET when $\theta=6$. 


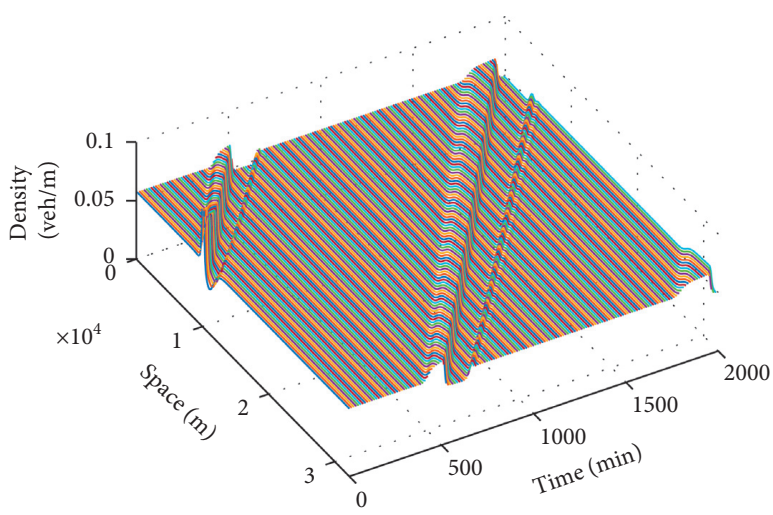

(a)

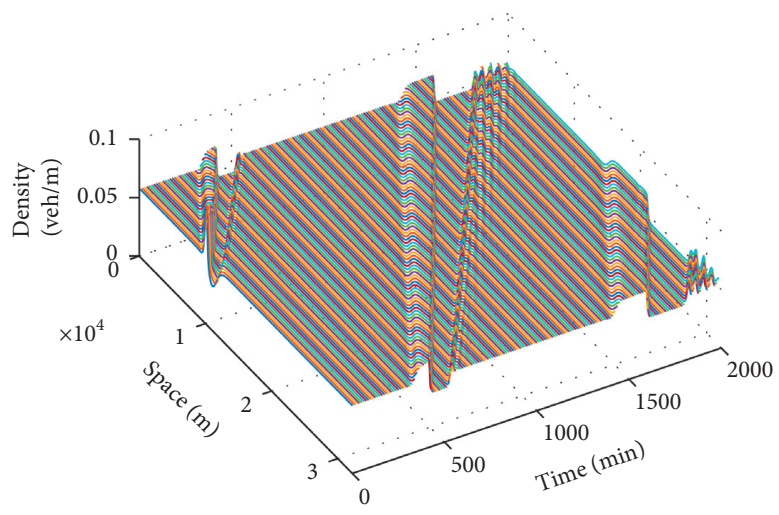

(c)

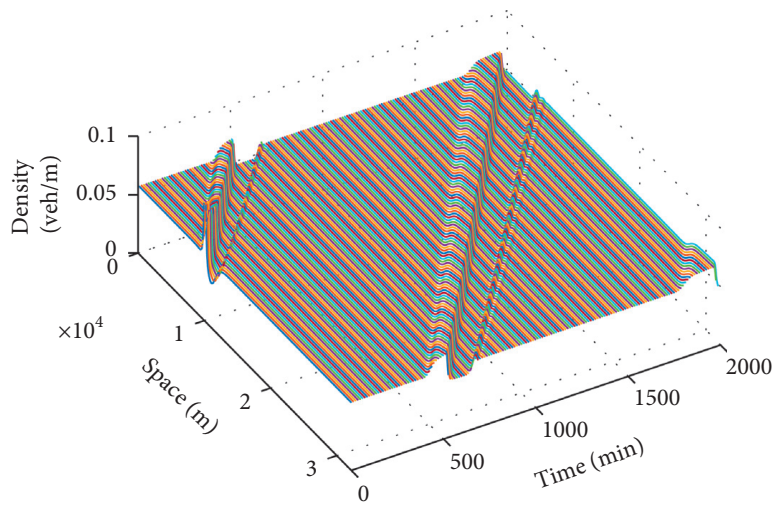

(e)

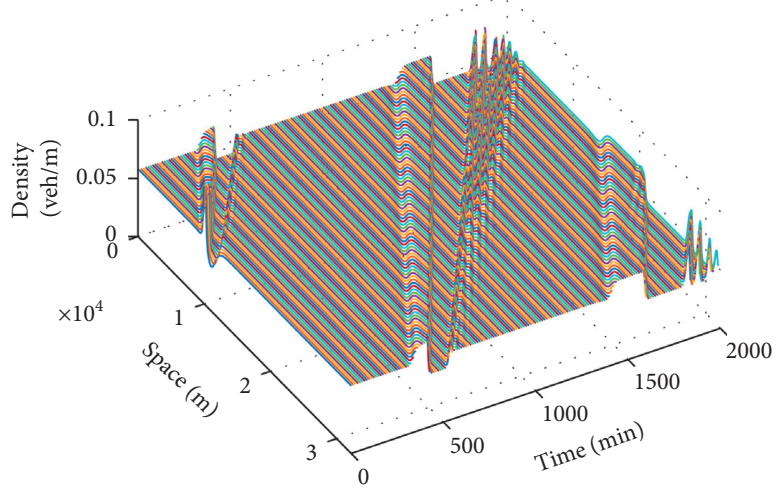

(g)

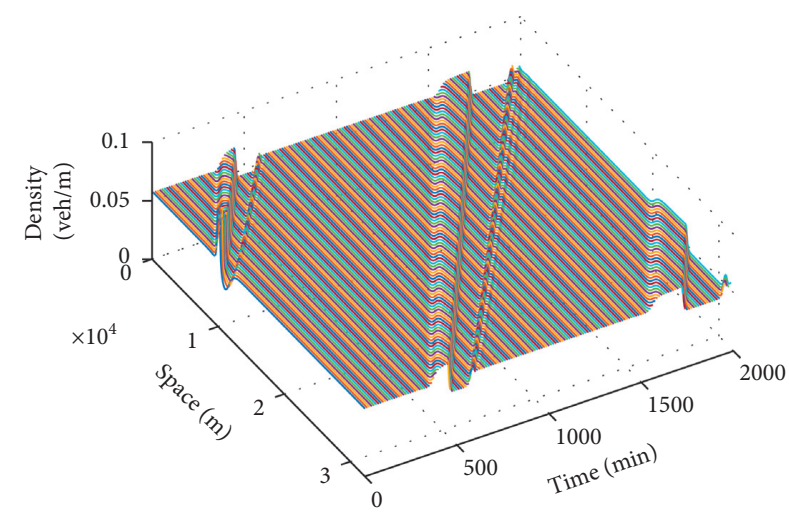

(b)

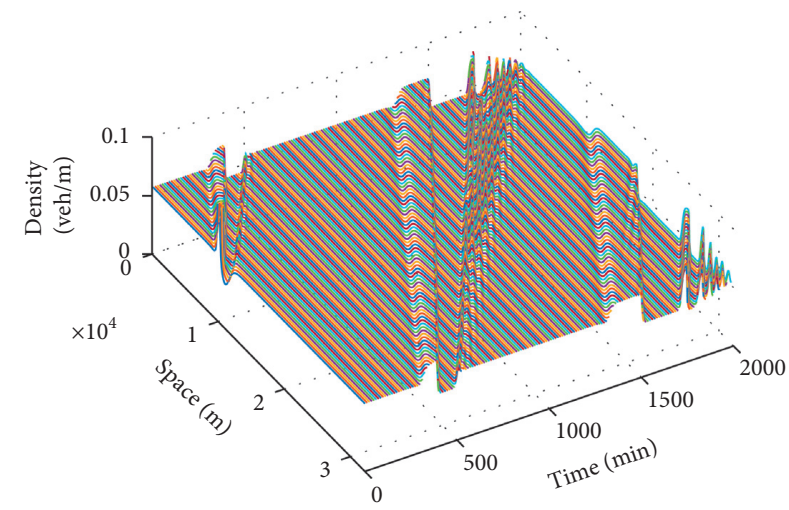

(d)

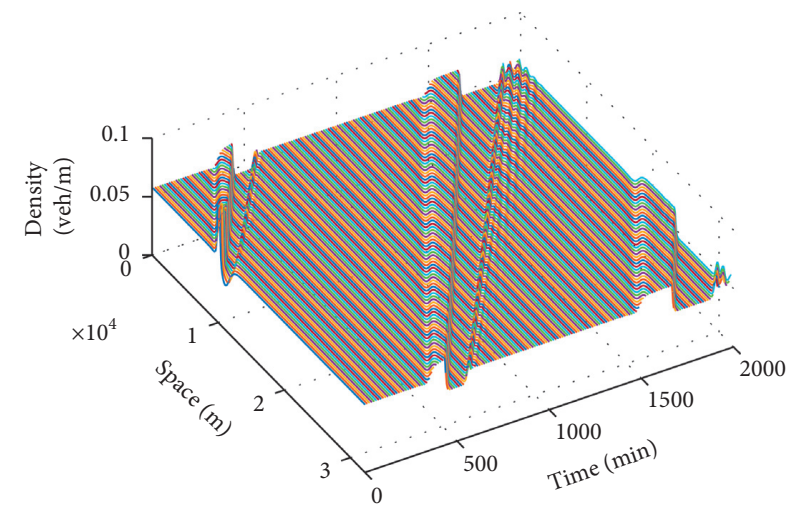

(f)

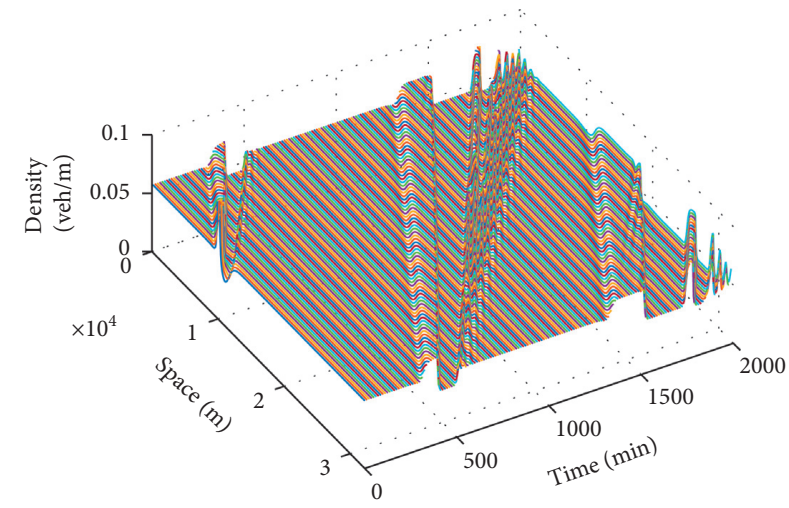

(h)

FIGURE 10: The evolution of traffic flow density wave in temporal and spatial during driver's timid driving downhill. (a) With ET when $\theta=0$. (b) With ET when $\theta=2$. (c) With ET when $\theta=4$. (d) With ET when $\theta=6$. (e) Without ET when $\theta=0$. (f) Without ET when $\theta=2$. (g) Without ET when $\theta=4$. (h) Without ET when $\theta=6$. 
with the model only considering driver's driving characteristics.

Figures 7 and 8 are the evolutionary charts of temporal and spatial density waves of traffic flow with driver's aggressive driving and timid driving considered, respectively, in the proposed continuum model on the uphill slope.

Figures $7(\mathrm{a})-7(\mathrm{~d})$ and Figures $8(\mathrm{a})-8(\mathrm{~d})$ are the evolution of new extended continuum model, while Figures $7(\mathrm{e})-7(\mathrm{~h})$ and Figures $8(\mathrm{e})-8(\mathrm{~h})$ do not consider the effect of electronic throttle angle. When the slope angle is fixed, the influence of electronic throttle angle can restrain the traffic congestion effectively and make traffic flow more stable. By comparing the change of traffic flow density wave from top to bottom, it can be concluded that the density wave fluctuation of the new macro model appears after the amplitude and frequency of the model which only considers the driver's driving characteristics. At the beginning, the slope is small, and the effect of small disturbance will be magnified, which will lead to the instability of traffic flow, and eventually the phenomenon of stop-and-go or traffic flow cluster appears. However, with the increase of slope and the passage of time, the effect of small disturbance will be reduced or even dissipated, and traffic flow will not stop at times or traffic flow cluster phenomenon. Therefore, the greater the gradient of the uphill road, the greater the role of traffic congestion suppression, thus the more stable the traffic flow. In addition, in Figures 7 and 8, it can be concluded that the stability of traffic flow becomes more and more stable with the increase of slope, and the stability of traffic flow can be effectively improved by considering electronic throttle factors. At the same time, the comparison between Figures 7 and 8 shows that the driver's radical driving is more helpful to alleviate traffic congestion and improve traffic flow stability than the driver's timid driving.

Figures 9 and 10 are the evolutionary charts of temporal and spatial density waves of traffic flow with driver's aggressive driving and timid driving considered, respectively, in the proposed macro traffic flow model on the downhill slope.

Figures 9(a)-9(d) and Figures10(a)-10(d) are density maps considering the effect of electronic throttle angle. Figures 9(e)-9(h) and Figures $10(\mathrm{e})-10(\mathrm{~h})$ are density maps without considering the effect of electronic throttle angle. When the slope angle is fixed, the comparison between $(\mathrm{a}-\mathrm{d})$ and $(\mathrm{e}-\mathrm{h})$ of Figures 9 and 10 can be obvious. The influence of electronic throttle angle can effectively restrain traffic congestion and make traffic flow more stable. By comparing the change of traffic flow density wave in $(a-d)$ and $(\mathrm{e}-\mathrm{h})$ of Figures 9 and 10 , it is obvious that the density wave fluctuation of the new traffic flow model occurs after the amplitude and frequency of the model only considering the driver's driving characteristics. At the beginning, the gradient of the ramp is small, the effect of small disturbance will not be amplified, and the inhibition effect on traffic congestion is obvious; thus the traffic flow tends to be stable. With the increase of slope angle, the effect of small disturbance becomes more and more obvious, and the traffic flow becomes more and more unstable, so the time- stop effect or traffic flow cluster effect will appear with the change of time. In addition, in Figures 9 and 10, it can be seen that the stability of traffic flow between aggressive driving and timid driving will become more and more unstable with the increase of slope, and considering electronic throttle factors can effectively improve the stability of traffic flow. At the same time, the comparison of Figures 9 and 10 shows that the driver's radical driving is more helpful to alleviate traffic congestion and improve traffic flow stability than the driver's timid driving in downhill driving.

\section{Conclusion}

Based on the classical car-following traffic flow model, this paper studies the change rule of traffic flow on sloping roads and proposes a car-following model on sloping roads. The driver's driving characteristics and the electronic throttle (ET) dynamics are introduced into the model. The new carfollowing model is transformed into a new continuum model by using the relationship between micro-variables and macro-variables. The stability condition of the new model is obtained by linear analysis theory; the KdV-Burgers equation solitary wave solutions for describing traffic flow change near the neutral stability curve are derived by using nonlinear analysis. According to the results of numerical simulation and experimental verification, the correctness of the model is verified. To sum up, we have reasonable reasons to draw conclusions from the following points. Firstly, in the course of driving on the ramp, radical driving can make shock wave and sparse wave smoother than timid driving, which is consistent with the theoretical analysis. Moreover, the impact of driver's radical driving and electronic throttle angle is a positive impact on shock wave and sparse wave; it is mainly reflected in the shock wave. In addition, the impact of radical driving and electronic throttle angle is obvious to help alleviate traffic congestion. That is to say, the new continuum model can smooth the wave front of traffic flow wave. Through theoretical analysis and numerical experiments, it is obvious that the traffic flow of aggressive driving is more stable than that of timid driving; that is, aggressive driving helps to alleviate traffic congestion when the slope angle of the ramp is unchanged whether on the uphill or downhill. When the driver's driving characteristics remain unchanged, on the uphill slope, the larger the slope angle is, the more stable the traffic flow is; on the downhill slope, the smaller the slope angle is, the more stable the traffic flow is. Last but not least, whether uphill or downhill, considering the ET effect can make the traffic flow more stable.

\section{Data Availability}

The data used to support the findings of this study are available from the corresponding author upon request.

\section{Conflicts of Interest}

The authors declare that there are no conflicts of interest regarding the publication of this paper. 


\section{Acknowledgments}

This work was supported by the Program of Humanities and Social Science of Education Ministry of China (Grant no. 20YJA630008), the Natural Science Foundation of Zhejiang Province, China (Grant no. LY20G010004), the National Key Research and Development Program of China-Traffic Modeling, Surveillance and Control with Connected \&Automated Vehicles (Grant no. 2017YFE9134700), and the K.C. Wong Magna Fund in Ningbo University, China.

\section{References}

[1] B. V. Arem, C. J. G. V. Driel, and R. Visser, "The impact of cooperative adaptive cruise control on traffic-flow characteristics," IEEE Transactions on Intelligent Transportation Systems, vol. 7, pp. 429-436, 2006.

[2] R. C. Carlson, I. Papamichail, M. Papageorgiou, and A. Messmer, "Optimal motorway traffic flow control involving variable speed limits and ramp metering," Transportation Science, vol. 44, no. 2, pp. 238-253, 2010.

[3] R. C. Carlson, I. Papamichail, and M. Papageorgiou, "Local feedback-based mainstream traffic flow control on motorways using variable speed limits," IEEE Transactions on Intelligent Transportation Systems, vol. 12, no. 4, pp. 1261-1276, 2011.

[4] T. Q. Tang, W. F. Shi, X. B. Yang, Y. P. Wang, and G. Q. Lu, “A macro traffic flow model accounting for road capacity and reliability analysis," Physica A: Statistical Mechanics and Its Applications, vol. 392, no. 24, pp. 6300-6306, 2013.

[5] H.-X. Ge, P.-J. Zheng, S.-M. Lo, and R.-J. Cheng, "TDGL equation in lattice hydrodynamic model considering driver's physical delay," Nonlinear Dynamics, vol. 76 , no. 1 , pp. 441-445, 2014.

[6] D. Helbing and B. Tilch, "Generalized force model of traffic dynamics," Physical Review E, vol. 58, no. 1, pp. 133-138, 1998.

[7] Z. P. Li, F. Q. Liu, and J. Sun, "A lattice traffic model with consideration of preceding mixture traffic information," Chinese Physics B, vol. 20, Article ID 088901, 2011.

[8] M. Bando, K. Hasebe, A. Nakayama, A. Shibata, and Y. Sugiyama, "Dynamical model of traffic congestion and numerical simulation," Physical Review E, vol. 51, no. 2, pp. 1035-1042, 1995.

[9] T.-Q. Tang, W.-F. Shi, H.-J. Huang, W.-X. Wu, and Z. Song, "A route-based traffic flow model accounting for interruption factors," Physica A: Statistical Mechanics and Its Applications, vol. 514, pp. 767-785, 2019.

[10] M. J. Lighthill and G. B. Whitham, "On kinematic waves. I. Flood movement in long rivers," Proceedings of the Royal Society of London Series A, vol. 229, pp. 281-316, 1955.

[11] M. J. Lighthill and G. B. Whitham, "On kinematic waves. II. A theory of traffic flow on long crowded roads," Proceedings of the Royal Society of London. Series A, vol. 229, pp. 317-345, 1955.

[12] P. G. Gipps, “A behavioural car-following model for computer simulation," Transportation Research Part B: Methodological, vol. 15, no. 2, pp. 105-111, 1981.

[13] H. X. Ge, S. Q. Dai, and L. Y. Dong, "Stabilization effect of traffic flow in an extended car-following model based on an intelligent transportation system application," Physical Review E, vol. 70, Article ID 066134, 2004.

[14] A. Kesting, M. Treiber, and D. Helbing, "General lanechanging model MOBIL for car-following models,"
Transportation Research Record: Journal of the Transportation Research Board, vol. 1999, no. 1, pp. 86-94, 2007.

[15] X. Zhao and Z. Gao, "A new car-following model: full velocity and acceleration difference model," The European Physical Journal B, vol. 47, no. 1, pp. 145-150, 2005.

[16] T. Q. Tang, H. J. Huang, and S. J. Huang, "A new car-following model with consideration of the traffic interruption probability," Chinese Physics B, vol. 387, pp. 975-983, 2009.

[17] G. H. Peng and L. H. Sun, "Multiple car-following model of traffic flow and numerical simulation," Chinese Physics B, vol. 57, pp. 5420-5430, 2009.

[18] K. Konishi, H. Kokame, and K. Hirata, "Coupled map carfollowing model and its delayed-feedback control," Physical Review E, vol. 60, no. 4, pp. 4000-4007, 1999.

[19] T. Tang, Y. Wang, X. Yang, and Y. Wu, "A new car-following model accounting for varying road condition," Nonlinear Dynamics, vol. 70, no. 2, pp. 1397-1405, 2012.

[20] W. X. Zhu and D. Z. Li, "A new car-following model for autonomous vehicles flow with mean expected velocity field," Physica A, vol. 492, pp. 2154-2165, 2018.

[21] W.-X. Zhu and H. M. Zhang, "Analysis of mixed traffic flow with human-driving and autonomous cars based on carfollowing model," Physica A: Statistical Mechanics and Its Applications, vol. 496, pp. 274-285, 2018.

[22] W.-X. Zhu, D. Jun, and L.-D. Zhang, "A compound compensation method for car-following model," Communications in Nonlinear Science and Numerical Simulation, vol. 39, pp. 427-441, 2016.

[23] H. Ou and T.-Q. Tang, "An extended two-lane car-following model accounting for inter-vehicle communication," Physica A: Statistical Mechanics and Its Applications, vol. 495, pp. 260-268, 2018.

[24] T.-Q. Tang, X.-F. Luo, J. Zhang, and L. Chen, "Modeling electric bicycle's lane-changing and retrograde behaviors," Physica A: Statistical Mechanics and Its Applications, vol. 490, pp. 1377-1386, 2018.

[25] T.-Q. Tang, J. Zhang, and K. Liu, “A speed guidance model accounting for the driver's bounded rationality at a signalized intersection," Physica A: Statistical Mechanics and Its Applications, vol. 473, pp. 45-52, 2017.

[26] Y. Sun, H. Ge, and R. Cheng, "An extended car-following model considering driver's memory and average speed of preceding vehicles with control strategy," Physica A: Statistical Mechanics and Its Applications, vol. 521, pp. 752-761, 2019.

[27] T. Wang, T.-Q. Tang, L. Chen, and H.-J. Huang, "Analysis of trip cost allowing late arrival in a traffic corridor with one entry and one exit under car-following model," Physica A: Statistical Mechanics and Its Applications, vol. 521, pp. 387398, 2019.

[28] G. H. Peng, X. H. Cai, B. F. Cao, and C. Q. Liu, "Non-lanebased lattice hydrodynamic model of traffic flow considering the lateral effects of the lane width," Physics Letters A, vol. 375, no. 30-31, pp. 2823-2827, 2011.

[29] Y.-R. Kang and D.-H. Sun, "Lattice hydrodynamic traffic flow model with explicit drivers' physical delay," Nonlinear Dynamics, vol. 71, no. 3, pp. 531-537, 2013.

[30] P. Redhu and A. K. Gupta, "Effect of forward looking sites on a multi-phase lattice hydrodynamic model," Physica A Statistical Mechanics, vol. 445, pp. 150-160, 2015.

[31] A. K. Gupta, S. Sharma, and P. Redhu, "Effect of multi-phase optimal velocity function on jamming transition in a lattice hydrodynamic model with passing," Nonlinear Dynamics, vol. 80, no. 3, pp. 1091-1108, 2015. 
[32] M. Zhao, L. H. Sun, and C. Tian, "Density waves in a lattice hydrodynamic traffic flow model with the anticipation effect," Chinese Physics B, vol. 21, pp. 619-624, 2012.

[33] C. Jiang, R. Cheng, and H. Ge, "An improved lattice hydrodynamic model considering the "backward looking" effect and the traffic interruption probability," Nonlinear Dynamics, vol. 91, no. 2, pp. 777-784, 2018.

[34] C. X. Ma, W. Hao, R. C. He et al., "Distribution path robust optimization of electric vehicle with multiple distribution centers," PLoS One, vol. 13, Article ID e0193789, 2018.

[35] C. X. Ma, W. Hao, F. Q. Pan, and W. Xiang, "Road screening and distribution route multi-objective robust optimization for hazardous materials based on neural network and genetic algorithm," PLoS One, vol. 13, Article ID e0198931, 2018.

[36] R. Cheng and Y. Wang, "An extended lattice hydrodynamic model considering the delayed feedback control on a curved road," Physica A: Statistical Mechanics and Its Applications, vol. 513, pp. 510-517, 2019.

[37] C. T. Jiang, R. J. Cheng, and H. X. Ge, "Mean-field flow difference model with consideration of on-ramp and offramp," Physica A, vol. 513, pp. 465-467, 2019.

[38] R. Jiang, Q.-S. Wu, and Z.-J. Zhu, "A new continuum model for traffic flow and numerical tests," Transportation Research Part B: Methodological, vol. 36, no. 5, pp. 405-419, 2002.

[39] L. H. Sun and G. H. Sun, "A viscous continuum traffic flow model with consideration of the coupling effect for two-lane freeways," Chinese Physics B, vol. 18, pp. 3724-3735, 2009.

[40] D. Vikram, P. Chakroborty, and S. Mittal, "Exploring the behavior of LWR continuum models of traffic flow in presence of shock waves," Procedia-Social and Behavioral Sciences, vol. 104, pp. 412-421, 2013.

[41] T. Nagatani, "Modified KdV equation for jamming transition in the continuum models of traffic," Physica A: Statistical Mechanics and Its Applications, vol. 261, no. 3-4, pp. 599-607, 1998.

[42] R. J. Cheng, F. X. Liu, and H. X. Ge, “A new continuum model based on full velocity difference model considering traffic jerk effect," Nonlinear Dynamics, vol. 89, pp. 1-11, 2017.

[43] R. Cheng, H. Ge, and J. Wang, "KdV-Burgers equation in a new continuum model based on full velocity difference model considering anticipation effect," Physica A: Statistical Mechanics and Its Applications, vol. 481, pp. 52-59, 2017.

[44] R. Cheng, H. Ge, and J. Wang, "An extended macro traffic flow model accounting for multiple optimal velocity functions with different probabilities," Physics Letters A, vol. 381, no. 32, pp. 2608-2620, 2017.

[45] Q. T. Zhai, H. X. Ge, and R. J. Cheng, "An extended continuum model considering optimal velocity change with memory and numerical tests," Physica A, vol. 490, pp. 774785, 2018.

[46] R. J. Cheng, H. X. Ge, and J. F. Wang, "The nonlinear analysis for a new continuum model considering anticipation and traffic jerk effect," Applied Mathematics and Computation, vol. 332, pp. 493-505, 2018.

[47] J. W. Payne and M. L. Braunstein, "Preferences among gambles with equal underlying distributions," Journal of Experimental Psychology, vol. 87, no. 1, pp. 13-18, 1971.

[48] M. Bando, K. Hasebe, K. Nakanishi, A. Nakayama, A. Shibata, and Y. k. Sugiyama, "Phenomenological study of dynamical model of traffic flow," Journal De Physique I, vol. 5, no. 11, pp. 1389-1399, 1995.

[49] B. Tilch and D. Helbing, "Evaluation of single vehicle data in dependence of the vehicle-type, lane, and site," Traffic and Granular Flow, vol. 99, pp. 333-338, 1999.
[50] R. Jiang, Q. S. Wu, and Z. Zhu, "Full velocity difference model for a car-following theory," Physical Review E, vol. 64, pp. 017-101, 2001.

[51] R. Cheng, H. Ge, and J. Wang, "An extended continuum model accounting for the driver's timid and aggressive attributions," Physics Letters A, vol. 381, no. 15, pp. 1302-1312, 2017.

[52] S. C. Yang, M. Li, Y. Lin, and T. Q. Tang, "Electric vehicle's electricity consumption on a road with different slope," Physica A: Statistical Mechanics and Its Applications, vol. 402, pp. 41-48, 2014.

[53] J. Zhou, Z.-K. Shi, and J.-L. Cao, "An extended traffic flow model on a gradient highway with the consideration of the relative velocity," Nonlinear Dynamics, vol. 78, no. 3, pp. 1765-1779, 2014.

[54] J. Chen, Z. Shi, Y. Hu, L. Yu, and Y. Fang, "An extended macroscopic model for traffic flow on a highway with slopes," International Journal of Modern Physics C, vol. 24, no. 9, p. 1350061, 2013.

[55] Y. Li, L. Zhang, S. Peeta, X. He, T. Zheng, and Y. Li, “A carfollowing model considering the effect of electronic throttle opening angle under connected environment," Nonlinear Dynamics, vol. 85, no. 4, pp. 2115-2125, 2016.

[56] T. Sidwell, G. Richards, K. Casleton et al., "Optically accessible pressurized research combustor for computational fluid dynamics model validation," Aiaa Journal, vol. 44, no. 3, pp. 434-443, 2006.

[57] K. S. Richards, "Simulation of flow geometry in a riffle pool stream," Earth Surface Processes \& Landforms, vol. 3, pp. 345-354, 2010.

[58] R. Jiang, Q. Wu, and Z. Zhu, "A new dynamics model for traffic flow," Chinese Science Bulletin, vol. 46, no. 4, pp. 345-348, 2001.

[59] M. Herrmann and B. S. Kerner, "Local cluster effect in different traffic flow models," Physica A: Statistical Mechanics and Its Applications, vol. 255, no. 1-2, pp. 163-188, 1998.

[60] B. S. Kerner and P. Konhauser, "Cluster effect in initially homogeneous traffic flow," Physical Review E, vol. 48, pp. 2335-2338, 1993. 